\title{
Stochastic Asymmetric Blotto Game Approach for Wireless Resource Allocation Strategies
}

\author{
Su Fong Chien ${ }^{*}$, Charilaos C Zarakovitis ${ }^{\dagger \S}$, Qiang $\mathrm{Ni}^{\S}$ and Pei Xiao
}

\begin{abstract}
The development of modellings and analytical tools to structurise and study the allocation of resources through noble user competitions become essential, especially considering the increased degree of heterogeneity in application and service demands that will be cornerstone in future communication systems. Stochastic asymmetric Blotto games appear promising to modelling such problems, and devising their Nash equilibrium (NE) strategies by anticipating the potential outcomes of user competitions. In this regard, this paper approaches the generic energy efficiency problem with a new stochastic asymmetric Blotto game paradigm to enable the derivation of joint optimal bandwidth and transmit power allocations by setting multiple users to compete in multiple auction-like contests for their individual resource demands. The proposed modelling innovates by abstracting the notion of fairness from centrally-imposed to distributed-competitive, where each user's pay-off probability is expressed as quantitative bidding metric, so as, all users' actions can be interdependent, i.e., each user attains its utility given the allocations of other users, which eliminates the chance of lowvalued carriers not being claimed by any user, and, in principle, enables the full utilisation of wireless resources. We also contribute by resolving the allocation problem with low complexity using new mathematical techniques based on Charnes-Cooper transformation, which eliminate the additional coefficients and multipliers that typically appear during optimisation analysis, and derive the joint optimal strategy as a set of linear singlevariable functions for each user. We prove that our strategy converges towards a unique, monotonous and scalable NE, and examine its optimality, positivity and feasibility properties in detail. Simulation comparisons with relevant studies confirm the superiority of our approach in terms of higher energy efficiency performance, fairness index and quality-of-service provision.
\end{abstract}

Index Terms - Charnes-Cooper transformation, competitive game, energy efficiency, green communications, Nash equilibrium, radio resource scheduling, stochastic asymmetric Blotto games.

\section{INTRODUCTION}

In radio resource allocation, there is a fundamental trade-off between efficiency and fairness metrics. Proportional games are shown to strike a balance between these metrics by maximising sum of utility functions, introducing, thereby, a centrally-imposed kind of fairness, which appears to be unfair from the individual user perspective as the respective Nash

* Mathematical Modelling Lab, MIMOS Berhad, Technology Park Malaysia, Kuala Lumpur, 57000, Malaysia (sf.chien@mimos.my)

$\dagger$ Institute of Information and Telecommunications, NCSR Demokritos, Athens, 153 10, Greece (c.zarakovitis@iit.demokritos.gr)

$\S$ School of Computing and Communications, Lancaster University, Bailrigg, LA1 4WA, UK (\{q.ni, c.zarakovitis1\}@lancaster.ac.uk)

$\$$ Institute for Communication Systems, University of Surrey, Guildford, GU2 7XH, UK (p.xiao@surrey.ac.uk)

Manuscript received October 2018,; revised May 2019; accepted August 2019. This work was supported in part by the projects EU-H2020 IoRL No.761992, MIS SYNTELESIS No.5002521, and UK EPSRC EP/R001588/1. (Corresponding Author: Qiang Ni) equilibriums (NEs) are not results of users' competition [1]. A solution is to let users being responsible for their own actions by providing them fictional budgets that can be spent on-demand towards receiving fractions of resources through engaging in competitive games, which facilitates abstracting the notion of fairness from centrally-imposed to distributed, with more degrees of freedom for resource control and potentially better performance improvements.

Game competitions have a natural interpretation in terms of lobbying for actions, and in their broader context can be viewed as extensions of the Colonel Blotto game of the auction/warfare theory [2]-[4]. Epigrammatically, the Blotto game was firstly approached in 1921 as a two-player twocontest zero-sum game [2], extended in 1950 to a two-player over an $N$-contest play [3], and consolidated in 2017 as a $K$-player $N$-contest game [4]. Interestingly, the latest $\langle K, N\rangle$ version of Blotto game offers a variety of probabilistic mechanisms for assigning each user with asymmetric (different) attributes and goals, and determining the winner of each contest stochastically through auction-like bidding process for maximising a majority value across all contests, which makes each user's pay-off utility a continuous function, thus, results in a unique strategy equilibrium allocation of user's bids across the $N$ contests. By contrast, in the proportional (noncompetitive) game, such as the General Lotto game, Duelling game, Stackelberg leadership, etc. [5], users are assigned with symmetric (identical) attributes and goals, which yields to a canonical deterministic mixed strategy that typically includes auctions for each user to maximise its total expected payoff at each contest altruistically, thus, converges towards a multiplicity of possible (non unique) equilibria. On this basis, the stochastic asymmetric Blotto game can potentially better realise the heterogeneity of mobile users' needs and the characteristics of their wireless environments, which are always dynamically changing, and in turn, better coordinate the systematic distribution of radio resources by anticipating the results of users' competitions.

The practical significance of stochastic asymmetric Blotto games is paramount for evolving the decision-making logic of next-generation communication systems by shifting from centrally-imposed to distributed-competitive notions of fairness, where the term "users" (players) can expand from mobile users to complete campus-wide networks, service providers, smart verticals, or even virtual network functions (VNFs), network nodes, etc., and wireless services (contests) can draw on enhanced Mobile BroadBand (eMBB) profiles with transformational Ultra Reliable Low Latency Communications (URLLC) and massive Machine Type Communications 
(mMTC) applications (3GPP Releases 15 \& 16). For example, stochastic asymmetric Blotto game logic can jointly integrate eMBB, URLLC and mMTC services into single multi-network profile when applied on, e.g., (i) cognitive radios (CRs) by enabling unlicensed mobile users to simultaneously compete for spare licensed carriers in both good and bad channel conditions, which ensures that no resources are wasted [6][7], (ii) mobile ad-hoc networks (MANETs) by deliberating both best-effort and poorly-treated nodes, which maximises the number of active routes and, thus, device connectivity [8], (iii) software-defined networking (SDN) by hierarchising the coexisting network nodes according to their instantaneous capacity, which helps to self-configure the traffic load balancing rules in runtime [9]-[10], (iv) multi-hop networks by distributing hops that are close as well as away from the gateways, which maximises the number of data flows and, thus, the network throughput overall [11], (v) Fog computing by anticipating the benefit of each Edge-Cloud device considering both free and congested computation/storage resources [12], (vi) network sharing by enabling multiple mobile operators with their networks deployed in the same area to collaborate and maximise instantaneous service-critical requests, while competing to improve their profits [13]-[14], and so on.

\section{A. Open Challenges and Research Contributions}

Although the rich literature dealing with the application of game theory to resource allocation problems, the stochastic asymmetric Blotto game has yet been carefully studied in the context of wireless communications. The open research challenges are summarised below.

- Most game theoretical problems are optimised with respect to (w.r.t) either bandwidth or transmit power operands, while joint allocation solutions are limited, e.g., [6]-[14], [19]-[20].

- Current optimisation utilities include the system sum-rate and/or sum-power, e.g., [6]-[7], [13], [19]-[20], while more inclusive objectives like the sum energy efficiency (sum$E E$ ) have yet been investigated, which can enable newer and better versions of user experiences [21]-[30].

- The utility pay-off probability is commonly expressed as qualitative biding metric to facilitate maximising users' bids at most profitable carriers, e.g., [5]-[7], [18]-[20], which compromises the efficiency-versus-fairness trade-off as lowbudgeted users may win less or even no carriers than high-budgeted users, while low-valued carriers may not be claimed by any user.

- Most attempts draw on deterministic NE strategies for the two-user two-contest Blotto game, e.g., [5]-[20], however, in practical systems the number of users and contests is much higher (tens or hundreds), which calls for broader multi-user multi-contest setting where the respective heterogeneity can be better captured through stochastic NE strategies.

- Existing optimisation analysis relies on dual programming that reduces the solution accuracy due to the uncontrollable duality gap [23]-[27], requires rather complex searching processes to obtain the sub-gradients of each possible optimal point, and cannot guarantee convergence as an optimal point may have more than one sub-gradient or even non [30]-[31].
This work contributes by addressing the above open challenges with unique game-theoretic design and solution method to the radio resource allocation problem inferring to the hypothesis that users' behaviour during competitions is acquisitive, while in proportional games imperative, and therefore, any efficiency-versus-fairness enhancements acquired stochastically are fundamentally superior over that acquired deterministically. The key innovation draws on maximising the system sum- $E E$ w.r.t both bandwidth and transmit power operands by introducing a new stochastic asymmetric Blotto game, where $K$ users bid fictional budgets across $N$ spectrum carriers simultaneously to compete for as many bandwidth portions as possible at each carrier - not for the most profitable carriers as done conventionally. Such game approach requires radically different problem formulation and solution manipulation, which we carry out by redefining the user pay-off probability from qualitative to quantitative biding metric based on the, so-called, competition for the majority [15]-[16] that derives the optimal bandwidth allocations, while enhancing the profile of low-budgeted users and low-valued carriers, which combats resource scarcity by minimising the chances that some users not to be allocated with carriers, and some carriers not to be claimed by any user. As long as spectrum is fully utilised, we apply Charnes-Cooper transformation (CCT) [17] on the generic quasi-convex sum- $E E$ problem to obtain its equivalent concave for what we derive the optimal transmit power allocations using standard (non-dual) Lagrangian optimisation analysis with robust mathematical method to render the intermediate CCT coefficients and Lagrangian multipliers. We show that our method resolves the optimal allocation points for each user as low-complex single-variable functions, and converges to a unique NE state that is positive, monotonous and scalable as long as the signal-to-noise-rate (SNR) of each carrier exceeds a certain minimum threshold, which specifies the Pareto boundary of the wireless system. We then prove that the proposed NE strategy complies with the non-degenerate constraint qualification (NDCQ) condition, and converges towards strict optimal points, which are also global due to the convexity of the CCT transformed problem. Finally, we adopt Long Term Evolution-Advanced (LTE-A) channel configuration and similar simulation setting with the relevant research studies in [21]-[30] to evaluate our findings in terms of sum- $E E$ performance, computational time, fairness index and quality-of-service (QoS) provision, and provide discussions to justify the observed discrepancies in comparison to those studies.

The remainder of this paper is structured as follows. Section II presents the system model and formulates the optimisation problem under study. Section III designs the stochastic Blotto game and derives the joint optimal strategy by explaining its key features. Section IV proves the existence and uniqueness of asymmetric NE in the proposed strategy and specifies the Pareto boundaries of the system. Section V details the implementation process, computational complexity and level of optimality of the solution. Section VI provides simulation evaluations and comparisons with relevant schemes, with Section VII to conclude the paper. 


\section{SYSTEM MODEL AND PROBLEM FORMULATION}

\section{A. System Model}

The examined system aims at maximising the sum- $E E$ by deriving dynamically the allocations of bandwidths $\left\{b_{k}^{n}\right\}$ and transmit powers $\left\{p_{k}^{n}\right\}$ of each user $k \in\{1,2, \ldots, K\}$ over each spectrum carrier $n \in\{1,2, \ldots, N\}$. The channel state information of the $k$-th user over the $n$-th carrier depends on the channel gain ${ }^{1}\left|h_{k}^{n}\right|^{2}=\Upsilon^{n} \cdot f_{k}^{n} \cdot\left(d_{k}\right)^{-\alpha^{n}}$, which, in turn, depends on (i) the distance $\left(d_{k}\right)^{-\alpha^{n}}$ between user $k$ and $\mathrm{BS}$, where $\alpha^{n}$ is the path-loss exponent of carrier $n$, (ii) the Rayleigh random variable $f_{k}^{n}$ that captures the fading of the $(k, n)$ radio link, and (iii) the path-loss constant $\Upsilon^{n} \cong\left(\frac{v^{n}}{4 \pi}\right)^{2}$, where $v^{n}$ is the wavelength of carrier $n$. On this basis, we define the signal-to-noise ratio (SNR) of user $k$ over carrier $n$ by $S N R_{k}^{n}=\frac{p_{k}^{n} \cdot\left|h_{k}^{n}\right|^{2}}{b_{k}^{n} \cdot N_{0}}$, where $N_{0}$ is the noise power spectral density of the additive white Gaussian noise ${ }^{2}$. Accordingly, we formulate the sum-rate as $r_{T x}=\sum_{k=1}^{K} r_{k}$, with $r_{k}=\sum_{n=1}^{N}\left(b_{k}^{n} \cdot r_{k}^{n}\right), \forall k$, the rate allocated to user $k$, and $r_{k}^{n}=\log _{2}\left(1+S N R_{k}^{n}\right), \forall k, n$, the rate allocated to user $k$ on carrier $n$. Furthermore, to ensure QoS satisfaction we bound the data rate $r_{k}$ of each user $k$ with its minimum throughout requirement $q_{k}$, i.e.,

$$
r_{k} \geq q_{k}, \forall k \text {. }
$$

Also, to assure that carriers do not overflow their physical capacity, we bound (i) the total bandwidth of each carrier with maximum load ${ }^{3} w^{n}$ that can be handled in carrier $n$, i.e., [21]

$$
\sum_{k=1}^{K} b_{k}^{n} \leq w^{n}, \forall n,
$$

and (ii) the total power transmitted from the BS to all users over all carriers with the available (supplied) power at the BS $p_{\max }$, i.e.,

$$
p_{T x}=\sum_{k=1}^{K} \sum_{n=1}^{N} p_{k}^{n} \leq p_{\max } .
$$

As a common practice in the literature, the generic sum$E E$ optimisation problem of the considered system can be formulated as [21]-[26], [27]-[30]

$$
\max _{b_{k}^{n}>0, p_{k}^{n} \geq 0} E E=\frac{r_{T x}}{\zeta \cdot p_{T x}+p_{c}} \quad \text { s.t. (1), (2), (3), }
$$

where $p_{c}$ denotes the circuit power consumed at the BS's transmitter and the distant receiver for each $(k, n)$ radio link due to signal processing, battery backup, etc., while coefficient $\zeta$ captures the power consumption due to the BS's amplifier inefficiency, feeder losses, etc.

\section{B. Problem Formulation}

Problem (4) aims at deriving bandwidth and transmit power allocations, so as to maximise the sum- $E E$ subject to the QoS, bandwidth and transmit power allocation constraints. A main contribution of this work is to enable the property of compet-

\footnotetext{
${ }^{1}$ Notation $|\cdot|^{2}$ indicates absolute value of complex numbers, e.g., $|x|^{2}=$ $x[x]^{T}$, with $[\cdot]^{T}$ the complex conjugate transpose.

${ }^{2}$ The considered channel can be extended to more realistic model, e.g. imperfect channel state information under fast fading channel, bandwidth broken in discrete chunks, etc. [27]. However, the main scope of this work is to analyse the design, solution and properties of the proposed game approach Therefore, we choose a channel model similar to [1], [6]-[14], [20]-[22], which includes the reference channel dynamics of LTE-A, while simplifies presenting the scope of this work. Also, our work can be easily extended from singleto multi-cell scenarios if regarding the inter-cell interference as noise.

${ }^{3}$ The maximum load $w^{n}$ depends on the carrier's frequency, e.g., carriers in mmWave bands have higher $w^{n}$ than carriers in radio frequency (RF) bands.
}

itive fairness in problems in the form of (4) for capturing the heterogeneity among multiple users of different attributes and goals, while boosting the sum- $E E$ performance through diversity gains due to the multi-user competition. Also, we will not compromise the complexity of the optimisation problem (4) by considering additional fairness constraints as, e.g., in [6][14], [20], [23]. In contrast, we will simplify the problem with stochastic asymmetric Blotto game logic and CCT transformation to derive the optimal bandwidth and power allocations with low complexity. Particularly, we consider that each user $k$ is allocated with a total bandwidth (budget) $B_{k}^{\max } \geq \sum_{n=1}^{N} b_{k}^{n}$ to be spent on $N$ carriers (contests) ${ }^{4}$. Users compete with each other by bidding their resources between carriers at each time slot simultaneously, while the user who acquires the highest amount of resources to a specific carrier wins this carrier. In this regard, we describe the stochastic asymmetric Blotto game by $\mathbf{B}=\left\langle K,\left\{B_{k}\right\}, u_{k}\left(\mathbf{b}_{k}, \mathbf{p}_{k}\right)\right\rangle$, where $K$ is the index set for the bidders (users), $B_{k}=\left[0, B_{k}^{\max }\right]$ the bandwidth allocation strategy set, and $u_{k}\left(\mathbf{b}_{k}, \mathbf{p}_{k}\right)$ the user utility function, with $\mathbf{b}_{k}=\left(b_{k}^{1}, \ldots, b_{k}^{n}, \ldots, b_{k}^{N}\right)$ and $\mathbf{p}_{k}=\left(p_{k}^{1}, \ldots, p_{k}^{n}, \ldots, p_{k}^{N}\right)$ the bandwidth and the corresponding transmit power allocation vectors for the $k$-th user, respectively. The spectrum sharing process of problem (4) can be then described by

$$
\begin{aligned}
& \text { (B1): } \max _{b_{k}^{n}>0} u_{k}\left(\left(b_{k}, p_{k}\right),\left(b_{k^{\prime}}, p_{k^{\prime}}\right)\right) \\
& \text { s.t. }(\mathrm{C} 1): \sum_{k=1, k \neq k^{\prime}}^{K} b_{k}^{n} \leq w^{n}, \forall n,
\end{aligned}
$$

where utility $u_{k}\left(\left(b_{k}, p_{k}\right),\left(b_{k^{\prime}}, p_{k^{\prime}}\right)\right)=\sum_{n=1}^{N}\left(\operatorname{Pr}_{k}^{n} \cdot w^{n} \cdot r_{k}^{n}\right)$, $\forall k, k^{\prime}$, is the stochastic pay-off of each user in the game, $\left(b_{k^{\prime}}, p_{k^{\prime}}\right)$ the pay-off of user $k^{\prime}$, and $\operatorname{Pr}_{k}^{n}$ the probability of user $k \neq k^{\prime}$ to win carrier $n$. Note that the utility in (5) does not measure the actual throughput a user can achieve at the end of the game. Instead, it provides a game utility to evaluate how users bid their resources (budgets) to different carriers (contests) based on their expected throughout (interest) on those carriers. This utility will be quantified later in the actual throughput to calculate the optimal sum- $E E$.

Furthermore, given that numerator and denominator of the sum- $E E$ objective function in (4) is concave and affine, respectively, legitimises applying CCT technique to track the fractional problem (4) with its equivalent concave-linear problem for adaptive power distribution [17]. That is, considering the variable transformation $y_{k}^{n}=p_{k}^{n} \cdot t$ with $t=$ $\frac{1}{\sum_{k=1}^{K} \sum_{n=1}^{N}\left(\zeta \cdot p_{k}^{n}\right)+p_{c}}>0$ such that $\sum_{k=1}^{K} \sum_{n=1}^{N}\left(\zeta \cdot y_{k}^{n}\right)+t \cdot p_{c}=1$, problem (4) can be transformed into its CCT equivalent problem given below.

$$
\begin{array}{ll}
\text { (B2) }: & \max _{y_{k}^{n}, t \geq 0} \sum_{k=1}^{K} \sum_{n=1}^{N} \eta_{0} \cdot t \cdot b_{k}^{n} \cdot \log _{2}\left(1+\frac{y_{k}^{n} \cdot\left|h_{k}^{n}\right|^{2}}{t \cdot b_{k}^{n} \cdot N_{0}}\right) \\
\text { s.t. } & (\mathrm{C} 2): \sum_{k=1}^{K} \sum_{n \in \Omega_{k}} y_{k}^{n} \leq t \cdot p_{\max }, \forall k, n, \\
& \text { (C3) }: t \cdot\left(\sum_{n=1}^{N} b_{k}^{n} \cdot \log _{2}\left(1+\frac{y_{k}^{n} \cdot\left|h_{k}^{n}\right|^{2}}{t \cdot b_{k}^{n} \cdot N_{0}}\right)-q_{k}\right)=0, \\
& \text { (C4) }: \sum_{k=1}^{K} \sum_{n=1}^{N}\left(\zeta \cdot y_{k}^{n}\right)+t \cdot p_{c}-1=0 .
\end{array}
$$

\footnotetext{
${ }^{4}$ For game competitions to end up with a feasible solution, players should express their needs through bidding common and valuable resources, like, e.g., real money, stocks, soldiers, etc. In the context of wireless communications, the most common and valuable resource is bandwidth [6]-[14], [20], [23]. The practicality of assuming that a user possesses bandwidth for biding purposes can be perceived upon expanding the notion of mobile users to network operators and service providers, which contest to attract more clients by bidding more bandwidth to improve the services required by those clients.
} 
Problem (6) aims at maximising the sum- $E E$ (B2) w.r.t the CCT operands $y_{k}^{n}$ and $t$, subject to total downlink transmit power $(\mathrm{C} 2)$, minimum user QoS requirements $(\mathrm{C} 3)$, and the CCT constraint (C4) for global optimality. Note that problem (6) is concave-linear since its objective (B2) and constraint (C3) are concave, while constraints (C2) and (C4) are affine w.r.t $y_{k}^{n}$ and $t$. Also, recall problem (5), which is linear w.r.t $b_{k}^{n}$ as both its objective (B1) and constraint $(\mathrm{C} 1)$ are affine. Therefore, the initial (fractional) sum- $E E$ problem (4) can be decoupled into two linear problems written as (a) maximise (B1) w.r.t $b_{k}^{n}$ s.t. (C1), and (b) maximise (B2) w.r.t $y_{k}^{n}$ and $t$ s.t. (C2)-(C4). The set of feasible solutions over $\left(b_{k}^{n}, y_{k}^{n}\right)$ in (4) is the union of the set of feasible solutions of the linear problems (a) and (b) as mentioned previously, and can be represented by the intersection of the double cone $\left\{b_{k}^{n} \mid(\mathrm{C} 1), b_{k}^{n}>0\right\} \cup\left\{y_{k}^{n}=\right.$ $\left.p_{k}^{n} \cdot t \mid(\mathrm{C} 2)-(\mathrm{C} 3), t>0\right\}$ inside $\mathbb{R}^{(K \times N)+1}$ (due to the additional parameter $t$ ), with the half-space (C4), i.e., $\mathbf{y}^{T} \cdot \zeta+\mathbf{t}^{T} \cdot p_{c}=1$. The intersection of this double cone with the half-space is a union of two convex sets w.r.t $\left\{b_{k}^{n}, p_{k}^{n}\right\}$ in an affine subspace of $K \times N$ dimensions. In this regard, we can draw this intersection by applying convex optimisation analysis on problems (5) and (6) to derive the optimal solutions over $b_{k}^{n}$ and $p_{k}^{n}$, respectively, which will be elaborated in Section III.

\section{JOINT OPTIMAL BLOTTO GAME STRATEGY FOR RADIO RESOURCE ALLOCATION}

This section derives the joint optimal bandwidth and transmit power allocations of the proposed stochastic asymmetric Blotto game model incorporating the property of competitive fairness. Meanwhile, we highlight and discuss key features and contributions of the proposed solution.

\section{A. Gaming Strategy for Joint Optimal Bandwidth and Trans-} mit Power Scheduling

A main factor to impact the bandwidth allocation strategy in game $\mathbf{B}=\left\langle K,\left\{B_{k}\right\}, u_{k}\left(\mathbf{b}_{k}, \mathbf{p}_{k}\right)\right\rangle$ is probability $\operatorname{Pr}_{k}^{n}$ of the pay-off in (5), which has been conventionally expressed as qualitative biding metric assuming each user aims to maximise the aggregate value of its budgets at most profitable carriers, e.g., $\operatorname{Pr}_{k}^{n}=\frac{b_{k}^{n}}{\sum_{k=1}^{K} b_{k}^{n}}$ [5]-[7], [18]-[20]. This way, however, the bidding process may turn into purely egocentric (competition for endogenous sharing) rather than altruistic (competition for a majority value), which potentially violates fairness (i.e. lowbudgeted users are most likely to win much less carriers than high-budgeted users or even none), and/or waste resources (i.e. low-valued carriers may not be claimed by any user) [15][16]. Instead, we define $\operatorname{Pr}_{k}^{n}=\frac{b_{k}^{n}}{\sum_{n=1}^{N} b_{k}^{n}}$ as quantitative biding estimation metric in $u_{k}\left(\left(b_{k}, p_{k}\right),\left(b_{k^{\prime}}, p_{k^{\prime}}\right)\right)$ to encourage users maximising the aggregate value of their budgets at the majority of the carriers (not only at the most profitable carriers as done conventionally). Our scope is to enhance the profile of lowbudgeted users and low-valued carriers in the bidding process, which reduces the chance (i) some users not to be allocated with carriers, and (ii) some carriers not to be claimed by any user. Theorem 1 follows readily.

Theorem 1. Given problems (5) and (6) the stochastic asymmetric Blotto game is formulated as $\mathbf{B}=$ $\left\langle K,\left\{B_{k}^{*}\right\}, u_{k}\left(\mathbf{b}_{k}^{*}, \mathbf{p}_{k}^{*}\right)\right\rangle$, with the winning strategy $B_{k}^{*}$ of each user $k \in K$ to be specified by the allocation profile $\left(\mathbf{b}_{k}^{*}, \mathbf{p}_{k}^{*}\right)$, where the optimal bandwidth allocation vector $\mathbf{b}_{k}^{*}=\left(b_{k}^{1 *}, \ldots, b_{k}^{n *}, \ldots, b_{k}^{N *}\right)$ includes the individual optimal bandwidth allocation of user $k$ on carrier $n$ defined as

$$
b_{k}^{n *}=b^{n} \cdot \frac{\sqrt{r_{k}^{n *} \cdot \sum_{n^{\prime}=1, n^{\prime} \neq n}^{N} b_{k}^{n^{\prime} *}}}{\sum_{k^{\prime}=1}^{K} \sqrt{r_{k^{\prime}}^{n *} \cdot \sum_{n^{\prime}=1, n^{\prime} \neq n}^{N} b_{k^{\prime}}^{n^{\prime} *}}}, \forall k, n,
$$

$r_{k}^{n *}=\frac{1}{\ln (2)} \cdot\left[\ln \left(1+S N R_{k}^{n *}\right)-\frac{S N R_{k}^{n *}}{\left(1+S N R_{k}^{n *}\right)} \cdot\left(1+\beta_{k}^{n}\right)\right]$ and $\beta_{k}^{n}=\frac{b_{k}^{n *}}{\sum_{n^{\prime}=1}^{N} b_{k}^{n^{\prime} *}}>0$, and the corresponding optimal transmit power allocation vector $\mathbf{p}_{k}^{*}=\left(p_{k}^{1 *}, \ldots, p_{k}^{n *}, \ldots, p_{k}^{N *}\right)$ includes the individual optimal transmit power of user $k$ on carrier $n$ defined as

$$
\begin{aligned}
& p_{k}^{n *}=b_{k}^{n *} \cdot\left(\omega_{k^{\prime}} \gamma_{k} \cdot \frac{b_{k^{\prime}}}{b_{k}} \cdot \frac{\prod_{n=1}^{N}\left(\frac{\left|h_{k^{\prime}}^{n}\right|^{2}}{N_{0}}\right)^{\gamma_{k} \frac{b_{k^{\prime}}^{n *}}{b_{k}}}}{\prod_{n=1}^{N}\left(\frac{\left|h_{k}^{n}\right|^{2}}{N_{0}}\right)^{\frac{b_{k}^{n *}}{b_{k}}}}-\frac{N_{0}}{\left|h_{k}^{n}\right|^{2}}\right), \forall k, n, \\
& \omega_{k^{\prime}}{ }^{*}=\frac{\left(\frac{p_{c}}{\zeta \cdot b^{\prime} m \cdot K \cdot N}-\frac{N_{0}}{\left|h_{k^{\prime}}^{m}\right|^{2}}\right)}{W\left(\prod_{m=1}^{N}\left(\frac{\left|h_{k^{\prime}}^{m}\right|^{2}}{N_{0}}\right)^{\frac{k^{\prime}}{b_{k^{\prime}}^{*}}} \cdot\left(\frac{p_{c}}{\zeta \cdot b_{k^{\prime}}^{m *} \cdot K \cdot N}-\frac{N_{0}}{\left|h_{k^{\prime}}^{m}\right|^{2}}\right) \cdot e^{-1}\right)}, \gamma_{k}=\frac{q_{k}}{q_{k^{\prime}}}
\end{aligned}
$$

and $W(\cdot)$ the Lambert-W function.

Proof. The proofs of the optimal bandwidth and power allocation solution in (7) and (8) of Theorem 1 are presented in Appendix A and Appendix B, respectively.

\section{B. Features of the Proposed Gaming Strategy}

The proposed joint optimal game strategy in Theorem 1 comprises two main features. First, the optimal bandwidth $b_{k}^{n *}$ and transmit power $p_{k}^{n *}$ in (7) and (8), respectively, depend neither on the CCT coefficients $y_{k}^{n}$ and $t$, nor on Lagrangian optimisation multipliers, i.e. the multipliers $\xi_{k}^{*}, \lambda^{*}, \mu^{*}$, which correspond to constraints $(\mathrm{C} 2),(\mathrm{C} 3),(\mathrm{C} 4)$, respectively, and were used during the convex analysis in Appendix $\mathrm{A}$ and Appendix B. Instead, $b_{k}^{n *}$ and $p_{k}^{n *}$ have been rendered into functions of the known system parameters $p_{c}, \zeta, K, N, h_{k}^{n}$. As we discuss in Appendix B and confirm later through simulations in Section IV, such feature reduces significantly the implementation complexity of the proposed strategy, especially compared to dual approaches, where additional multipliers are commonly included in the optimal formulas [21]-[28], [31].

The second feature is that the optimal $b_{k}^{n *}$ and $p_{k}^{n *}$ in (7) and (8), respectively, demonstrate interdependence among users, i.e., $b_{k}^{n *}$ (or $p_{k}^{n *}$ ) of user $k$ on carrier $n$ depends on $b_{k}^{n *}$ (or $p_{k}^{n *}$ ) of user $k$ on carrier $n^{\prime}$. This is mainly due to our consideration of $\operatorname{Pr}_{k}^{n}$ as quantitative biding metric into $u_{k}\left(b_{k}^{n}, p_{k}^{n}\right)$, which allows outlining the NE of optimal bandwidth allocations, whereby all users are satisfied with the pay-off attained given the allocations of other users, that has yet been considered by previous studies. In general, acquiring NE solutions offers a predictable and stable outcome for games where multiple players with conflicting interests compete to reach a NE state, where no player wishes to deviate. Nevertheless, such NE state (i) may not be unique, (ii) may bound the system to certain SNR thresholds or (iii) may not even exist. In Section IV, we study the existence, uniqueness and boundary conditions 
of NE along with key properties of the proposed joint game strategy in Theorem 1.

\section{NASH EQUILIBRIUM AND PROPERTIES OF THE PROPOSED BLOTTO GAME STRATEGY}

This section examines the competitive fairness properties of the proposed joint optimal strategy in Theorem 1, and its convergence towards unique NE state.

\section{A. Existence and definition of NE in the Proposed Gaming Strategy}

To show that the proposed joint optimal strategy complies with the property of competitive fairness it is sufficient to prove that it converges towards an existing and unique NE state. We do so, by focusing on the marginal rate of substitutions (MRSs) to capture how much additional satisfaction a unit of resource $b_{k}^{n *}$ (or resource $b_{k}^{n^{\prime} *}$ ) can bring to user $k$, while maintaining the same level of (utility) satisfaction [33]. In Theorem 1, the MRSs of user $k$ are the slopes of bandwidth equations $b_{k}^{n *}$ in (7) of vector $\mathbf{b}_{k}^{*}$ of the winning strategy $B_{k}^{*}$ of each user $k$, which can be written in functional form as

$$
B_{k}^{*}=-b_{k}^{n *}+b^{n} \cdot \frac{\sqrt{r_{k}^{n *} \cdot \sum_{n^{\prime}=1, n^{\prime} \neq n}^{N} b_{k}^{n^{\prime} *}}}{\sum_{k^{\prime}=1}^{K} \sqrt{r_{k^{\prime}}^{n *} \cdot \sum_{n^{\prime}=1, n^{\prime} \neq n}^{N} b_{k^{\prime}}^{n^{\prime} *}}}=0 .
$$

In principle, MRSs are represented by the partial derivatives (elements) of the Jacobian matrix $J_{B_{k}^{*}}$ of the winning strategy $B_{k}^{*}$, and they are identical when $B_{k}^{*}$ converges to a NE state. This implies that if matrix $J_{B_{k}^{*}}$ is non-singular then, according to Implicit Function Theorem [37], MRSs are identical, and thus, NE exists (see e.g., [35] pp.128). Proposition 1 follows readily.

Proposition 1. The Jacobian matrix $J_{B_{k}^{*}}$ of $B_{k}^{*}$ in (9) is nonsingular for $S N R_{k}^{n *}>5.306 \mathrm{~dB}$.

Proof. The proof of Proposition 1 is presented in Appendix C.

Proposition 1 identifies the minimum SNR threshold as the Pareto system boundary above which the Blotto game $\mathbf{B}=$ $\left\langle K,\left\{B_{k}^{*}\right\}, u_{k}\left(\mathbf{b}_{k}^{*}, \mathbf{p}_{k}^{*}\right)\right\rangle$ in Theorem 1 can reach the NE state where

$$
\begin{aligned}
\forall k,\left(\mathbf{b}_{k}, \mathbf{p}_{k}\right) \in B_{k}^{*} & : u_{k}\left(\left(\mathbf{b}_{k}^{*}, \mathbf{p}_{k}^{*}\right),\left(\mathbf{b}_{-k}^{*}, \mathbf{p}_{-k}^{*}\right)\right) \\
& \geq u_{k}\left(\left(\mathbf{b}_{k}, \mathbf{p}_{k}\right),\left(\mathbf{b}_{-k}^{*}, \mathbf{p}_{-k}^{*}\right)\right),
\end{aligned}
$$

with $\left(\mathbf{b}_{-k}^{*}, \mathbf{p}_{-k}^{*}\right)$ to represent the strategy profiles of all users except user $k$. The boundary implies that each carrier's signal power should be at least 3.4-times higher than noise power, which corresponds to the worst case scenario for all carriers' conditions where users have no incentive to contest. In this situation, although the game can carry on, it will not reach the NE in (10). Note that the considered SNR threshold is lower than the minimum SNR levels set by LTE-A and WiFi standards ${ }^{5}$, which indicates that the proposed game strategy is likely to reach its NE in practical setting. Another outcome of

\footnotetext{
${ }^{5}$ In a typical LTE-A and/or WiFi setting the SNR varies from $40 \mathrm{~dB}$ (excellent signal and association) to $10 \mathrm{~dB}$ (no signal and association). Generally, a signal with an SNR value of $20 \mathrm{~dB}$ or more is recommended for data applications where as an SNR value of $25 \mathrm{~dB}$ or more is recommended for voice applications [21], [26].
}

Proposition 1 is that since $J_{B_{k}^{*}}$ is non-singular, the MRSs are identical for any point $\bar{b}$ that lays in the tangent space at the variety of $b_{k}^{n *}$ [33]. This indicates that the NE of $B_{k}^{*}$ remains same for any descriptive point of $b_{k}^{n *}$ such as the maximum, minimum, mean or actual value of $b_{k}^{n *}$, which allows to rewrite $r_{k}^{n *}$ in (7) as

$$
\begin{aligned}
\bar{r}_{k}^{n *} & =\frac{1}{\ln (2)} \cdot\left(\ln \left(1+\overline{S N R}_{k}^{n *}\right)-\frac{\overline{S N R}_{k}^{n *}}{\left(1+\overline{S N R}_{k}^{n *}\right)}\right. \\
& \left.\times\left(1+\frac{\bar{b}}{\sum_{n^{\prime}=1}^{N} b_{k}^{n^{\prime *}}}\right)\right), \quad \overline{S N R}_{k}^{n *}=\frac{\left.p_{k}^{n *} \cdot h_{k}^{n}\right|^{2}}{\bar{b} \cdot N_{0}} .
\end{aligned}
$$

In view that throughput is large enough in practice (i.e. $r_{k}^{n *}>>$ 1 usually in Mbps), $\frac{\overline{S N R}_{k}^{n *}}{\left(1+\overline{S N R}_{k}^{n *}\right)}<1$ and term $\frac{\bar{b}}{\sum_{n^{\prime}=1}^{N} b_{k}^{n^{\prime} *}}$ is in the order of $10^{-3}$ [21], [29], the rate $\bar{r}_{k}^{n *}$ in (11) can be approached as

$$
\begin{aligned}
\bar{r}_{k}^{n *}= & \frac{1}{\ln (2)} \cdot\left(\ln \left(1+\overline{S N R}_{k}^{n *}\right)-\frac{\overline{S N R}_{k}^{n *}}{\left(1+\overline{S N R}_{k}^{n *}\right)} \cdot(\underbrace{1+\underbrace{\frac{\bar{b}}{\sum_{n^{\prime}=1}^{N b_{k}^{n^{\prime}}}}}_{10^{-3}})}_{<1})\right. \\
& \approx \frac{1}{\ln (2)} \cdot\left(\ln \left(1+\overline{S N R}_{k}^{n *}\right)-1\right) \\
& =\log _{2}\left(\frac{1+\overline{S N R}_{k}^{n *}}{e}\right),
\end{aligned}
$$

with $\bar{b} \rightarrow\left\{b^{\text {max }}=\max \left\{b_{k}^{n^{\prime *}}\right\}, b^{\text {min }}=\min \left\{b_{k}^{n^{\prime} *}\right\}, b^{\text {mean }}=\right.$ $\left.\frac{1}{N} \sum_{n^{\prime}=1}^{N} b_{k}^{n^{\prime *}}\right\}$ and $e$ the Euler's constant. Due to its compact form, $\bar{r}_{k}^{n *}$ in (12) can be implemented much more readily than conventional $r_{k}^{n *}$ in (7), while (as shown later through simulations) the impacts of both expressions on sum- $E E$ are comparable. In summary, Proposition 1 yields the SNR boundary for the existence of asymmetric NE in strategy of Theorem 1, as well as a robust expression of user throughputs to speed up the implementation of scheduling process with the comparable sum- $E E$ performance.

\section{B. Uniqueness of NE in the Proposed Gaming Strategy}

To ensure the convergence of the game strategy in Theorem 1, it is important to show that its NE not only exists but it is also unique. We examine the uniqueness of NE based on principles of the fixed-point theory [37], where the time of a discrete-time model is divided into iterations, while during each iteration $t$ all users can act only once and remain static. By letting $\mathbf{b}_{k}^{*}(t)$ and $\mathbf{b}_{k}^{*}(t+1)$ denote the bandwidth allocation vectors of user $k$ at current and next iteration $t$ and $(t+1)$, respectively, and by recalling $B_{k}^{*}$ in (9) we rewrite the winning strategy of $k$-th user as

$$
\boldsymbol{f}\left(\mathbf{b}_{k}^{*}(t)\right):=\mathbf{b}_{k}^{*}(t+1)=b^{n} \cdot \frac{\sqrt{r_{k}^{n *} \cdot \sum_{n^{\prime}=1, n^{\prime} \neq n}^{N} b_{k}^{n^{\prime} *}(t)}}{\sum_{k^{\prime}=1}^{K} \sqrt{r_{k^{\prime}}^{n *} \cdot \sum_{n^{\prime}=1, n^{\prime} \neq n}^{N} b_{k^{\prime}}^{n^{\prime} *}(t)}},
$$

with $\boldsymbol{f}\left(\mathbf{b}_{k}^{*}(t)\right)=\left(\boldsymbol{f}_{1}\left(b_{k}^{n *}(t)\right), \ldots, \boldsymbol{f}_{k}\left(b_{k}^{n *}(t)\right), \ldots, \boldsymbol{f}_{K}\left(b_{k}^{n *}(t)\right)\right)$ the arbitrary set of the $k$-th user joint best response functions at iteration $t$. From (13) we see that the convergence of $\mathbf{b}_{k}^{*}(t+1)$ at the next iteration $(t+1)$ depends on the current state of $\boldsymbol{f}\left(\mathbf{b}_{k}^{*}(t)\right)$ at iteration $t$. However, it is yet ensured that the NE state acquired at iteration $t$ exists and is unique at iteration $t+1$, especially when there are changes in some of the bidding resources, e.g., if $\boldsymbol{f}_{k}\left(b_{k}^{n *}(t)\right) \gtrless \boldsymbol{f}_{k}\left(b_{k}^{n *}(t+1)\right)$. Therefore, we need to ensure that, regardless of the number of iterations, the $\mathrm{NE}$ exists and is unique even if a best 
response function in $\boldsymbol{f}\left(\mathbf{b}_{k}^{*}(t)\right)$ changes. According to Banach's [38] and Rosen's [39] fixed-point theorems and as shown in [4], [34], pp. 553, among others, if the best response 2 functions of a non-cooperative game intersect and are standard ${ }_{4}^{3}$ functions for all users, then they converge to a unique NE 5 state at each iteration. A function $\boldsymbol{f}: \mathbf{b}_{k}^{*} \rightarrow \mathrm{R}_{+}^{K}$ is said 6 to be standard if and only if it is jointly (i) positive,i.e., $\forall b_{k}^{n *} \in \mathbf{b}_{k}^{*} \Rightarrow \boldsymbol{f}\left(b_{k}^{*}\right)>0$, (ii) monotonous, i.e., $\forall b_{k}^{n *}, b_{k}^{n^{\prime} *} \in \mathbf{b}_{k}^{*}$, , $b_{k}^{n *} \leq b_{k}^{n^{\prime} *} \Rightarrow \boldsymbol{f}\left(b_{k}^{n *}\right) \gtrless \boldsymbol{f}\left(b_{k}^{n^{\prime} *}\right)$ (component-wise), and (iii) scalable, i.e., $\forall \rho>1, b_{k}^{n *} \in \mathbf{b}_{k}^{*} \Rightarrow \rho \cdot \boldsymbol{f}\left(b_{k}^{n *}\right)>\boldsymbol{f}\left(\rho \cdot b_{k}^{n *}\right)$. Proposition 2 follows readily.

Proposition 2. The joint best response functions of set $\boldsymbol{f}\left(\boldsymbol{b}_{k}^{*}(t)\right)$ in (13) are jointly positive, monotonous and scalable, and intersect to a fixed and unique NE, where $\boldsymbol{f}\left(\mathbf{b}_{k}^{*}(t)\right):=$ $\mathbf{b}_{k}^{*}(t+1), \forall k \in K$.

Proof. The proof of Proposition 2 is presented in Appendix D.

In physical terms, (i) the positivity property in Proposition 2 certifies game feasibility, i.e., for any initial $\left\{b_{k}^{n}(t)\right\}$ values, the best response functions $\left\{\boldsymbol{f}_{k}\left(b_{k}^{n *}(t)\right)\right\}$ will generate a ${ }_{24}^{23}$ feasible NE solution $\left\{b_{k}^{n *}(t), p_{k}^{n *}(t)\right\}$ in $\boldsymbol{f}\left(\mathbf{b}_{k}^{*}(t)\right)$ that is also admissible for all users in $\boldsymbol{f}\left(\mathbf{b}_{k}^{*}(t+1)\right)$, (ii) the monotonicity property ensures game performance, i.e., each successful iteration will produce NE points $\left\{\mathbf{b}_{k}^{n}(t+1)\right\}$ that are better than any previous points $\left\{\mathbf{b}_{k}^{n}(t)\right\}$, and (iii) the scalability property confirms game reliability, i.e., if user $k$ has a winning chance under strategy $\left\{\mathbf{b}_{k}^{*}, \mathbf{p}_{k}^{*}\right\}$, then user $k$ will have a higher winning chance when all resources scale up uniformly.

In summary, Section IV shows that the proposed strategy in Theorem 1 converges towards a unique NE state, which can exist above a certain SNR threshold. In such NE state, our strategy inherits all properties of competitive fairness as listed in, e.g., [4]-[16]. That is, (i) all users in the Blotto game $\mathbf{B}$ will adhere to the joint strategy if the knowledge of the bandwidth and transmit power allocations of other users is not available, but it is believed that the available budget will be allocated according to (7) and (8). (ii) The NE in $\mathbf{B}$ has a monotonic property where the user with the higher budget has a better chance of winning the targeted carriers. (iii) All users in $\mathbf{B}$ compete for carriers, where even a small amount of additional budget improves the chance of winning more carriers. (iv) All users in $\mathbf{B}$ tend to fully spend their budget to increase the chance of winning more carriers regardless of the conditions on each carrier.

\section{IMPLEMENTATION, COMPLEXITY AND OPTIMALITY OF THE PROPOSED STRATEGY}

This section discusses the implementation process with computational complexity of the Blotto-type game strategy for joint optimal bandwidth and transmit power scheduling. Meanwhile, we study the level of optimality of the proposed mathematical methodology.

\section{A. Implementation process and Computational Complexity of the Proposed Game Strategy}

The joint optimal solution in Theorem 1 implements using two loops as shown in the pseudo-code of Algorithm 1. The outer

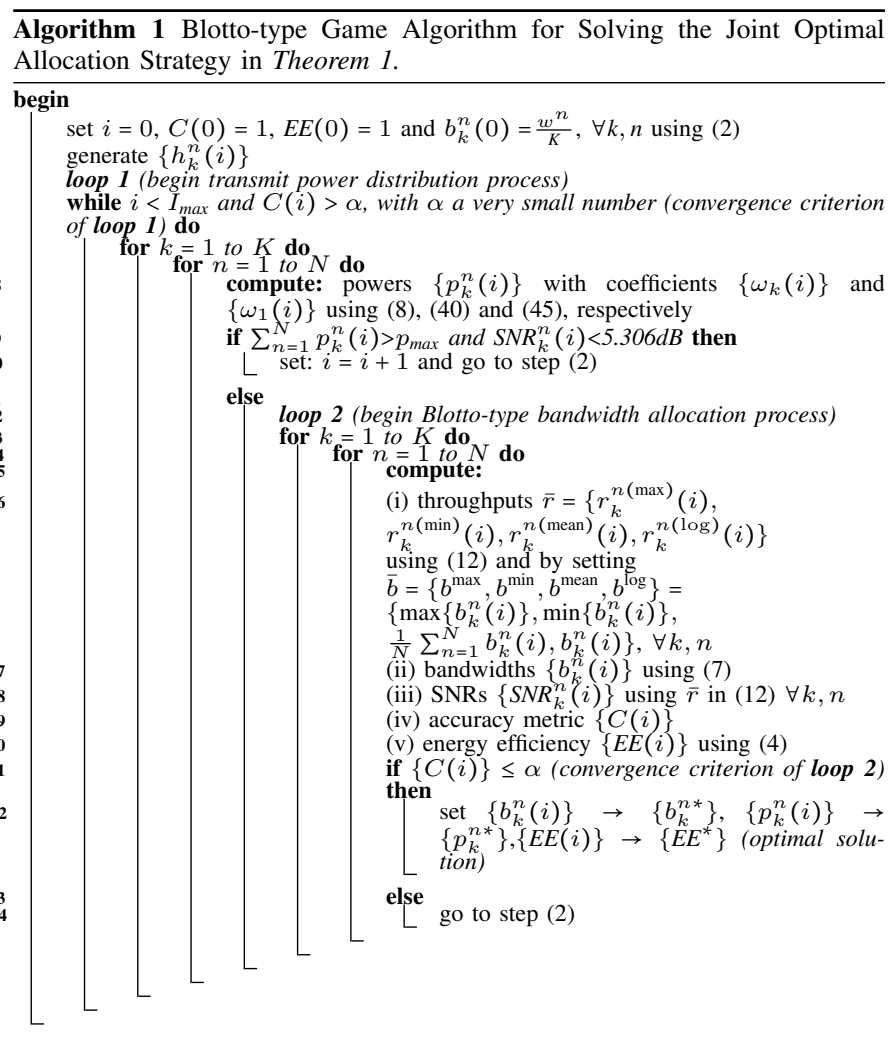

loop 1 is to define the optimal power $p_{k}^{n *}$ in (8), while the inner loop 2 the bandwidth $b_{k}^{n *}$ in (7). In Steps 1-3, Algorithm 1 loads the starting values for bandwidths $b_{k}^{n}(0)$ and energy efficiency $E E(0)$, while setting the stopping criterion $C(0)$ based on the metric $C(i)=\frac{\sqrt{\sum_{k=1}^{K} \sum_{n=1}^{N}\left(b_{k}^{n}(i+1)-b_{k}^{n}(i)\right)^{2}}}{\sqrt{\sum_{k=1}^{K} \sum_{n=1}^{N}\left(b_{k}^{n}(i)\right)^{2}}} \leq \alpha$, which is shown to effectively capture the predefined accuracy tolerance $\alpha$ w.r.t $\left\{b_{k}^{n *}(i), p_{k}^{n *}(i)\right\}$ at each iteration $i=0, \ldots, I_{\max }$, where $I_{\max }$ is the maximum number of iterations. With the initial values of $b_{k}^{n}(0), E E(0)$ and $C(0)$, loop 1 in Steps 4-11 computes the allocated powers $\left\{p_{k}^{n}(i)\right\}$ for each link $(k, n)$ at each iteration $i$ such that the feasibility criteria related to power constraint (3) and SNR threshold in Theorem 1 are both fulfilled. Then, in Steps 1225 loop 2 implements the Blotto game to update each user's bandwidths $\bar{b}$ with corresponding rates $\bar{r}$, system's energy efficiency $E E(i)$ and stopping criterion $C(i)$. The process terminates when either the number of iterations reaches the maximum threshold $I_{\max }$ (time criterion) or $C(i)$ becomes smaller than $\alpha$ (optimality criterion). Notice that due to NE states in (12), points $\left\{b_{k}^{n *}(i), p_{k}^{n *}(i)\right\}$ remain the same for any descriptive point $\bar{b}$ and therefore, in Step 17 of loop 2 we can choose between four measures for powers and bandwidths, i.e., $\left\{p_{k}^{n}\left(b^{\text {max }}, i\right), p_{k}^{n}\left(b^{\text {min }}, i\right), p_{k}^{n}\left(b^{\text {mean }}, i\right), p_{k}^{n}\left(b_{k}^{n}, i\right)\right\}$. As will be evidenced later with simulations, choosing $b^{\text {mean }}$ among those four options can bring about higher rate and sum- $E E$ performance.

Furthermore, recalling our analysis in Appendix A and Appendix B, and particularly Lemma 1 and equations (36)(45), which specifies that the utility $u_{k}\left(b_{n}^{n *}, p_{n}^{n *}\right)$ of each user $k$ is increasing, strictly concave and twice continuously 
differentiable in $b_{n}^{n *}$ and $p_{n}^{n *}$, the purely theoretical complexity for deriving $\left\{b_{n}^{n *}\right\}$ and $\left\{p_{n}^{n *}\right\}$ of the strategy in Theorem 1 increases linearly with the number of users and carriers, and it is in the order of $\mathcal{O}(K \cdot N)$ with $\mathcal{O}(\cdot)$ the Big-O notation. However in practice, the complexity of the game strategy in Theorem 1 depends on the computational complexity of Algorithm 1, which, in turn, depends on the complexity of the two considered loops. The complexity of loop 1 depends on the number of users and bandwidths and can be estimated by $\mathcal{O}\left(\alpha^{-1} \cdot K \cdot N\right)$ times at each iteration $i$. Similarly, loop 2 (Blotto game) requires $\mathcal{O}\left(\alpha^{-1} \cdot K \cdot N\right)$ times to reach a steady NE state. By denoting $T_{I_{\max }}$ as the time duration of each iteration, the total complexity of Algorithm 1 is in the order of $\mathcal{O}\left(T_{I_{\max }} \cdot I_{\max } \cdot \alpha^{-2} \cdot K \cdot N\right)$, which is much lower than complexities $\mathcal{O}\left(T_{I_{\max }} \cdot \alpha^{-2} \cdot(L+K \cdot N \cdot(\log N))\right)$ and $\mathcal{O}\left(2 \cdot T_{I_{\text {max }}} \cdot I_{\text {max }} \cdot \alpha^{-2} \cdot K \cdot N^{2} \cdot L\right)$ of the relevant schemes in [21] and [25], respectively, where $L$ is the number of optimisation constraints. This notable difference is because Dinkelbachtype schemes resolve the sum- $E E$ problem using dual programming, which includes time-consuming processes for the sequential search of the sub-gradients of each dual-optimal multiplier at each iteration (e.g. related to constraints (1)-(3)). Instead, our analysis relies on primary (non-dual) Lagrangian optimisation, where CCT approach stokes out from the final solution any extra (dual) multiplier and, therefore, Algorithm $l$ is independent from subgradient searching procedures.

\section{B. Optimality Level of the Proposed Gaming Strategy}

Notice that neither dual nor non-dual optimisation can provide any information about how optimal the final solution can be [31], [33]. That means the derived $\left\{b_{k}^{n *}, p_{k}^{n *}\right\}$ from Algorithm 1 (and the dual algorithms in [21] and [25]) may refer to either global optimal, local optimal or critical points of the sum- $E E$ problem (4). To ensure that $\left\{b_{k}^{n *}, p_{k}^{n *}\right\}$ are (at least) local optimal points of (4), it is sufficient to prove that the NDCQ condition for global optimality holds for the set

$$
\begin{aligned}
& C\left(g_{i}\left(y_{k}^{n}, t\right)\right)=\underbrace{\left\{\sum_{k=1}^{R} \sum_{n=1}^{N} y_{k}^{n}-t \cdot p_{\max }\right.}_{g_{0}\left(y_{k}^{n}, t\right)} \leq 0, \\
& \underbrace{t \cdot\left(\sum_{n=1}^{N} b_{k}^{n} \cdot \log _{2}\left(1+\frac{y_{k}^{n} \cdot\left|h_{k}^{n}\right|^{2}}{t \cdot b_{k}^{n} N_{0}}\right)-q_{k}\right)}_{g_{k}\left(y_{k}^{n}, t\right)} \geq 0, k=1, \ldots, K, \\
& \text { and } \underbrace{\sum_{k=1}^{K} \sum_{n=1}^{N}\left(\varsigma \cdot y_{k}^{n}\right)+t \cdot p_{c}-1}_{g_{K+1}\left(y_{k}^{n}, t\right)}=0\},
\end{aligned}
$$

which is determined by all CCT equivalent constraints $\left\{g_{i}\left(y_{k}^{n}, t\right)\right\}$ of problems (5) and (6) w.r.t points $\left\{y_{k}^{n *}, t^{*}\right\}$. The NDCQ optimality translates into three requisites: (i) the Jacobian matrix

$$
\begin{aligned}
G\left(g_{i}\left(y_{k}^{n}, t\right)\right)= & \left(\begin{array}{c}
\nabla g_{0}\left(y_{k}^{n}, t\right) \\
\vdots \\
\nabla g_{K+1}\left(y_{k}^{n}, t\right)
\end{array}\right) \\
= & \left(\begin{array}{ccc}
\frac{\partial g_{0}\left(y_{k}^{n}, t\right)}{\partial y_{1}^{1}} & \cdots & \frac{\partial g_{0}\left(y_{k}^{n}, t\right)}{\partial y_{k}^{n}} \\
\vdots & \ddots & \vdots \\
\frac{\partial g_{K+1}\left(y_{k}^{n}, t\right)}{\partial y_{1}^{1}} & \cdots & \frac{\partial g_{K+1}\left(y_{k}^{n}, t\right)}{\partial y_{k}^{n}}
\end{array}\right)
\end{aligned}
$$

of set $C\left(g_{i}\left(y_{k}^{n}, t\right)\right)$ (or the vector of gradients of $\left\{g_{i}\left(y_{k}^{n}, t\right)\right\}$ ) should be of rank $K+2$ (i.e. $K+1$ inequality and 1 CCT
TABLE I: Parameters for simulation of system and channel modelling

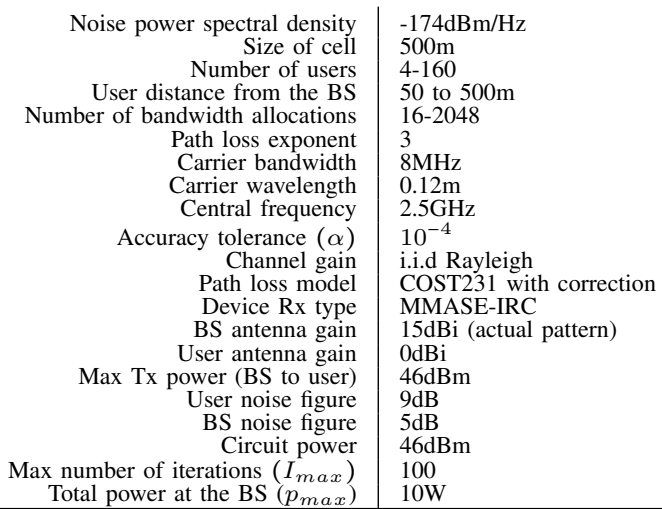

equality constraints w.r.t $\left\{y_{k}^{n *}, t^{*}\right\}$ ), (ii) there exists a nonzero vector $\boldsymbol{\psi}$ which resolves each constraint $g_{i}\left(y_{k}^{n}, t\right)$ of $G\left(g_{i}\left(y_{k}^{n}, t\right)\right)$ w.r.t $\left\{y_{k}^{n *}, t^{*}\right\}$, and (iii) constraints $\left\{g_{i}\left(y_{k}^{n}, t\right)\right\}$, $i=0, \ldots, K+1$ should be continuous and twice differentiable in $\mathbb{R}^{(K \cdot N)+1}$. Otherwise, if one or more NDCQ requisites are not fulfilled, constraints $(\mathrm{C} 1)-(\mathrm{C} 4)$ may be violated for some $\left\{y_{k}^{n *}, t^{*}\right\}$ points in $C\left(g_{i}\left(y_{k}^{n}, t\right)\right)$, hence our solution w.r.t $\left\{b_{k}^{n *}, p_{k}^{n *}\right\}$ can refer to critical points of the sum- $E E$ problem (4). Proposition 3 and Proposition 4 follow readily.

Proposition 3. The Jacobian matrix $G\left(g_{i}\left(y_{k}^{n}, t\right)\right)$ in (15) of set $C\left(g_{i}\left(y_{k}^{n}, t\right)\right)$ in (14) is of rank $K+2$ and there exists nonzero vector $\boldsymbol{\psi}$ such that $G\left(g_{i}\left(y_{k}^{n}, t\right)\right) \cdot \boldsymbol{\psi}^{T}=0$.

Proposition 4. Constraints $\left\{g_{i}\left(y_{k}^{n}, t\right)\right\}, i=0, \ldots, K+1$ of problems (5) and (6) are continuous and twice differentiable in $\mathbb{R}^{(K \cdot N)+1}$.

Proof. The proofs of Proposition 3 and Proposition 4 are presented in Appendix E.

Proposition 3 and Proposition 4 indicate that the NDCQ requisites hold for the solution of Theorem 1 hence, Algorithm 1 results in strict local optimal $\left\{b_{k}^{n *}, p_{k}^{n *}\right\}$ points. Also, since the CCT transformed problem (6) is convex, we can assert that points $\left\{b_{k}^{n *}, p_{k}^{n *}\right\}$ are global solutions of the initial problem (4) and the proposed Algorithm 1 converges towards the global optimal sum- $E E$ solution, i.e., $E E\left(r_{k}^{n *}\left(p_{k}^{n *}, b_{k}^{n *}\right)\right)$. We evaluate our schemes using simulations in Section VI.

\section{PERFORMANCE EVALUATIONS}

This section presents simulation results of the proposed solution and performs performance comparisons with most relevant resource scheduling schemes to examine the level of optimality and implementation complexity as well as the fairness, throughput/QoS and sum- $E E$ performances.

\section{A. System and Channel Simulation Modelling}

For the ease of presentation, we name our proposed solution in this paper as sum-EE optimisation based on Asymmetricallyfair Blotto game and CCT non-Dual approach (EE-ABCD). Also, for fair comparisons, we adopt the same modelling as LTE-A channel configuration used in [21]-[30]. These studies are most relevant to our work because they maximise the sum- $E E$ objective subject to QoS and carrier sharing 

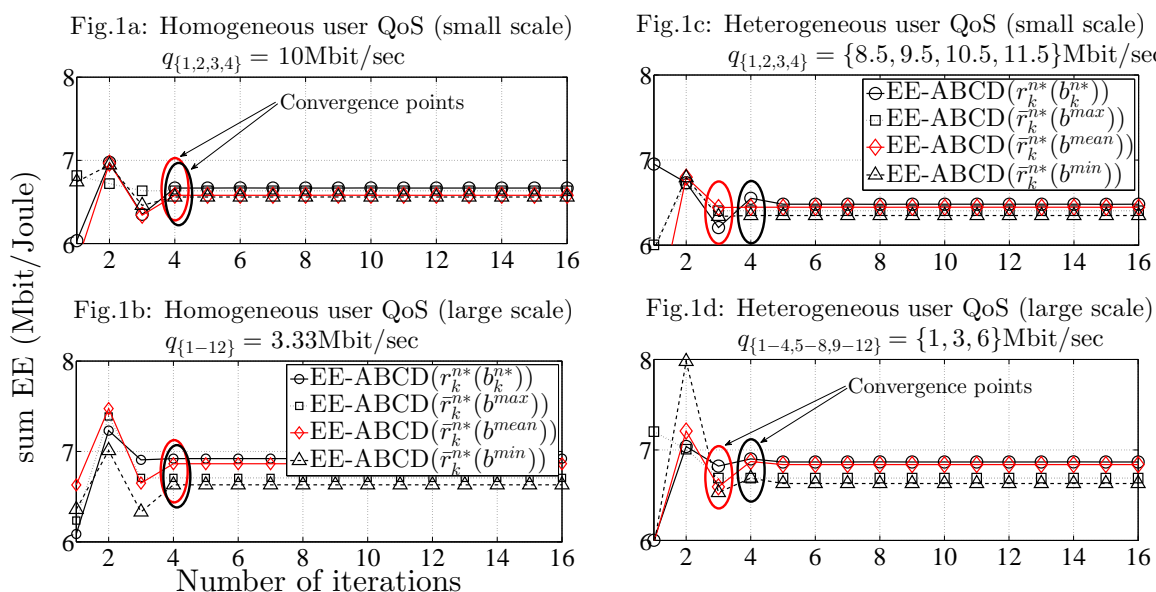

Fig.1d: Heterogeneous user QoS (large scale)

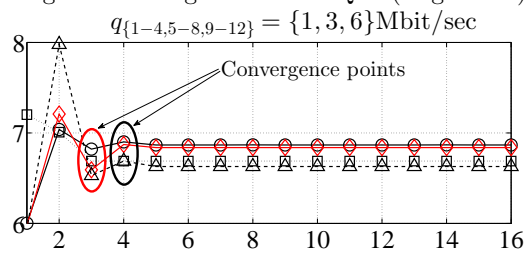

Fig. 1: Sum- $E E$ vs. number of iterations over various system scales and user heterogeneity: comparisons between the four versions of the proposed solution in Proposition 1.

constraints. However, our EE-ABCD differs because the sumEE optimisations in [21]-[23] rely on Dual-Optimal Sum-ofratios Optimisation (EE-DOSO) solution [31] with no game considerations. In addition, the Dinkelbach Algorithm-Type Approach (EE-DATA) in [24]-[30] is shown to derive the dual-optimal sum- $E E$ more accurately than EE-DOSO but at higher complexity. Also, we consider comparing with our previous game approach in [1], where we maximise the sumrate using Symmetrically-fair Nash bargaining Solution (RxTSNS) for joint bandwidth and power allocations in an effort to gain insights into the impact of proportional fairness ( $\mathrm{Rx}$ TSNS) versus the impact of competitive fairness (EE-ABCD) on system performance. The simulation parameters are given in Table I unless otherwise stated.

\section{B. Impact of Descriptive Bandwidth Allocations on sum-EE}

Fig.1 examines the level of optimality and computational complexity between all versions of EE-ABCD extracted by Proposition 1. In particular, we compare the performance of sum- $E E$ versus number of iterations between the expression of "actual" throughput $r_{k}^{n *}\left(b_{k}^{n *}\right)$ with "actual" bandwidth $b_{k}^{n *}$ in (7), and the "simplified" throughput $\bar{r}_{k}^{n *}(\bar{b})$ with "descriptive" bandwidths $(\bar{b}) \rightarrow\left\{b^{\text {max }}, b^{\text {min }}, b^{\text {mean }}\right\}$ in (12). We see that by using "actual" values of measures $b_{k}^{n *}$ attains higher sum- $E E$ than using "simplified" versions $\bar{b}$ but requires more time to converge, especially as the system scales up and/or user QoS heterogeneity increases. For example, with homogeneous QoS (Fig.1a-Fig.1b) EE-ABCD requires half the time to converge compared with heterogeneous QoS (Fig.1c-Fig.1d) (i.e. 3 iterations instead of 6 iterations, respectively), while in both cases performances are comparable (i.e. $0.03 \mathrm{Mbit} / \mathrm{Joule}$ difference in average). Interestingly, using mean bandwidth points $\left(\bar{r}_{k}^{n *}\left(b^{\text {mean }}\right)\right)$ results in almost similar sum- $E E$ with "actual" $\left(r_{k}^{n *}\left(b_{k}^{n *}\right)\right)$ (i.e. $0.02 \mathrm{Mbit} / \mathrm{Joule}$ difference in average) and converges much faster regardless of the system scale and heterogeneity (i.e. in Fig1.c-Fig.1d the sum $E E\left(\bar{r}_{k}^{n *}\left(b^{\text {mean }}\right)\right)$ converges in 3 iterations only). In conclusion, using the simplified expression of mean bandwidth $\left(\bar{r}_{k}^{n *}\left(b^{\text {mean }}\right)\right)$ in (12) attains the best optimality-vs-complexity trade-off among all versions of EE-ABCD.

\section{Evaluations on sum-EE and Computational Complexity}

Fig.2 expands the results of Fig.1 in order to compare between the sum- $E E$ performances of the proposed EE-ABCD and the relevant schemes EE-DOSO, EE-DATA and Rx-TSNS. For this experiment, we consider an additional algorithmic approach as reliable metric for global optimality of the original sum- $E E$ problem (4), namely exhaustive search (ES) [1], [21],
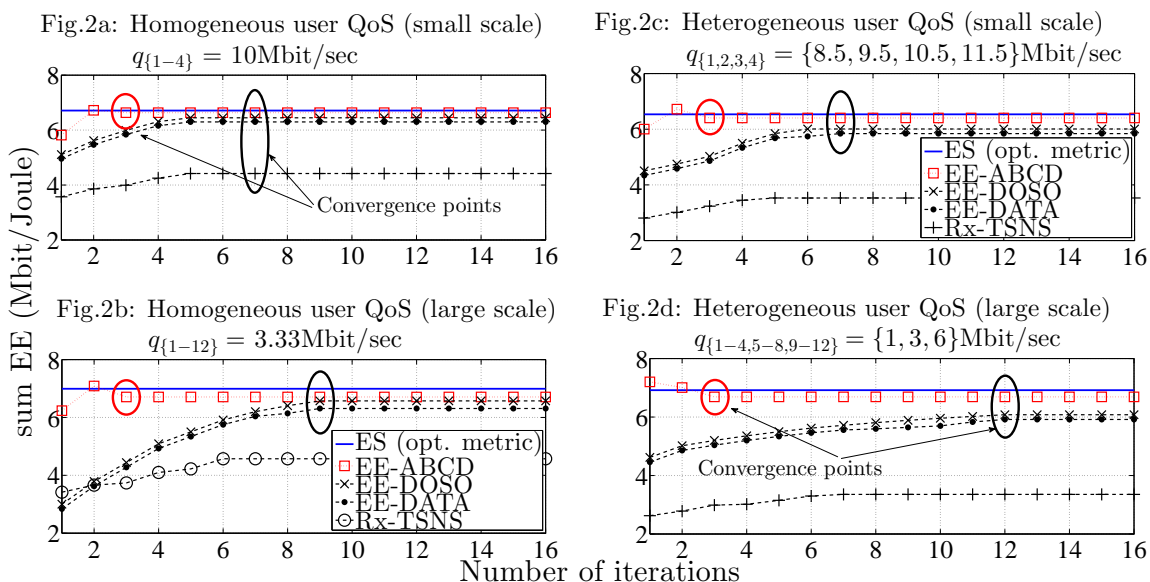

Fig.2d: Heterogeneous user QoS (large scale)

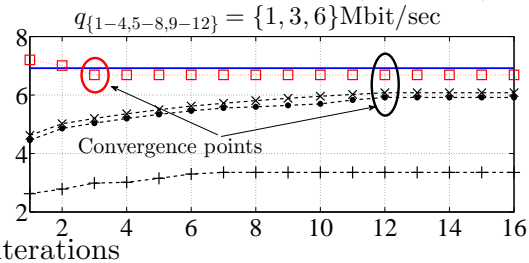

Fig. 2: Sum- $E E$ vs. number of iterations over various system scales and user heterogeneity: comparisons between the proposed EE-ABCD and relevant EE-DOSO, EE-DATA and Rx-TSNS. 


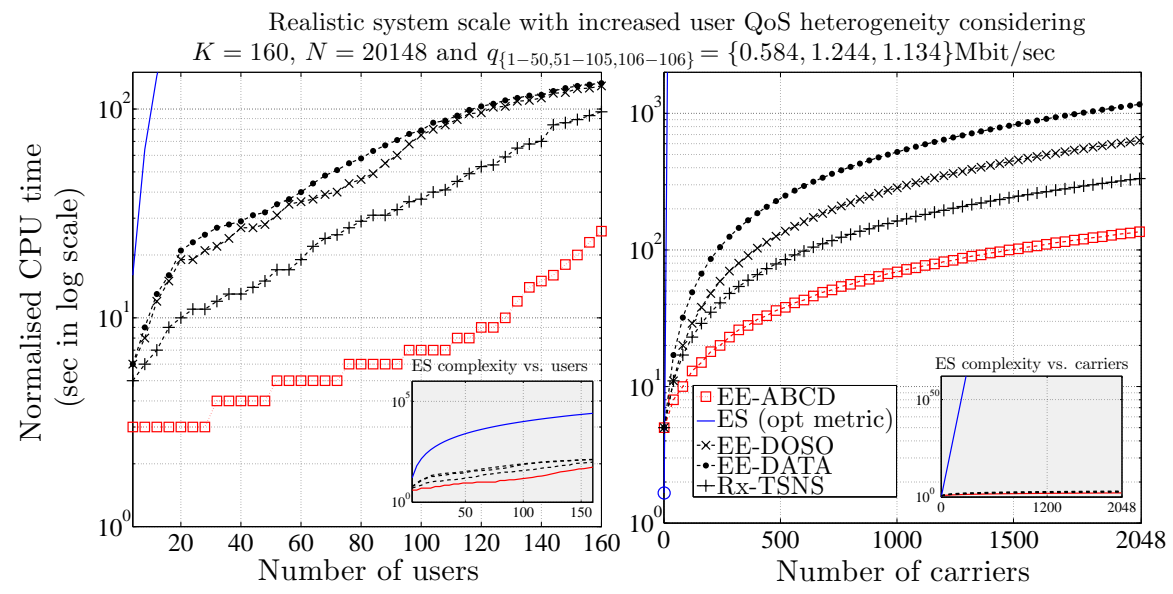

Fig. 3: Normalised CPU time vs. number of users and number of carriers in large scale system: comparisons on implementation complexity between the proposed EE-ABCD and relevant EE-DOSO, EE-DATA and Rx-TSNS.

[25], [28]. Note that due to the particularly high complexity of the ES (i.e. $\mathcal{O}\left(K^{N}\right)$ ), we present its solutions independently from the number of iterations. We see that EE-ABCD attains sum- $E E$ almost similar to ES and considerably higher than EE-DOSO, EE-DATA and Rx-TSNS. This is because the space $\left\{b_{k}^{n} \mid(\mathrm{C} 1), b_{k}^{n}>0\right\} \cup\left\{y_{k}^{n}=p_{k}^{n} \cdot t \mid(\mathrm{C} 2)-(\mathrm{C} 3), t>0\right\}$, where EEABCD determines the optimal points $\left\{b_{k}^{n *}, p_{k}^{n *}\right\}$, postulates the complete fulfilment of both QoS and transmit power constraint (1) and (3), respectively, otherwise NE states could never be reached and sum- $E E$ would rebate. In contrast, the corresponding spaces in EE-DOSO and EE-DATA depend on the weak duality that holds for either the QoS or transmit power constraint hence, some of the "actual" optimal points $\left\{b_{k}^{n *}, p_{k}^{n *}\right\}$ are excluded leading to lower performances, especially when the system scales up and/or user heterogeneity increases (see e.g. [26] pp. 76-77 for more details). Note that Rx-TSNS achieves the lowest sum- $E E$ than EE-DOSO and EE-DATA since it maximises throughput instead of energy efficiency. Furthermore, EE-ABCD in Fig.2a-Fig.2d converges in 3 iterations only, while EE-DOSO, EE-DATA and Rx-TSNS require from 7 up to 12 iterations. Such complexity difference is clearer in Fig.3, which considers realistic system scale of 2048 carriers and 160 users with increased QoS heterogeneity. Indeed, EE-ABCD converges in $28 \mathrm{sec}$ and $137 \mathrm{sec}$, while all other schemes require more than $100 \mathrm{sec}$ and $320 \mathrm{sec}$ to converge under full user and carrier load, respectively. This is because the implementation time of EE-ABCD is practically linear w.r.t both $K$ and $N$, and it is mainly dependent on the predefined accuracy tolerance $\alpha$, since the optimal bandwidths (7) in Theorem 1 have been decoupled between the $N \gg K$ system carriers. On the contrary, sub-gradient searching make EE-DOSO and EE-DATA to take longer to converge. In conclusion, EE-ABCD achieves higher sum- $E E$ in significantly less time than all the other examined schemes, especially in practical setting.

\section{Evaluations on Fairness and QoS Provision}

Fig.4 illustrates how fairly each scheme allocates the radio resources to system users by relying on the measure socalled Fairness Index $F I=\frac{\left(\sum_{k=1}^{K}\left(\frac{r_{k}}{q_{k}}\right)\right)^{2}}{K \cdot \sum_{k=1}^{K}\left(\frac{r_{k}}{q_{k}}\right)^{2}}$, where $F I=1$ indicates perfectly fair resource allocation, while $F I=0$ totally opportunistic scheduling [32]. For this experiment, we assume that ES's bandwidth assignments are fixed such that ES can serve as metric for perfect fairness provision. Our intention is to examine the ability of each scheme to distribute the available resources (i.e. $b_{k}^{n}, p_{k}^{n}$ and $p_{\max }$ ) in a way where (i) either all users are satisfied under resource sufficiency, or

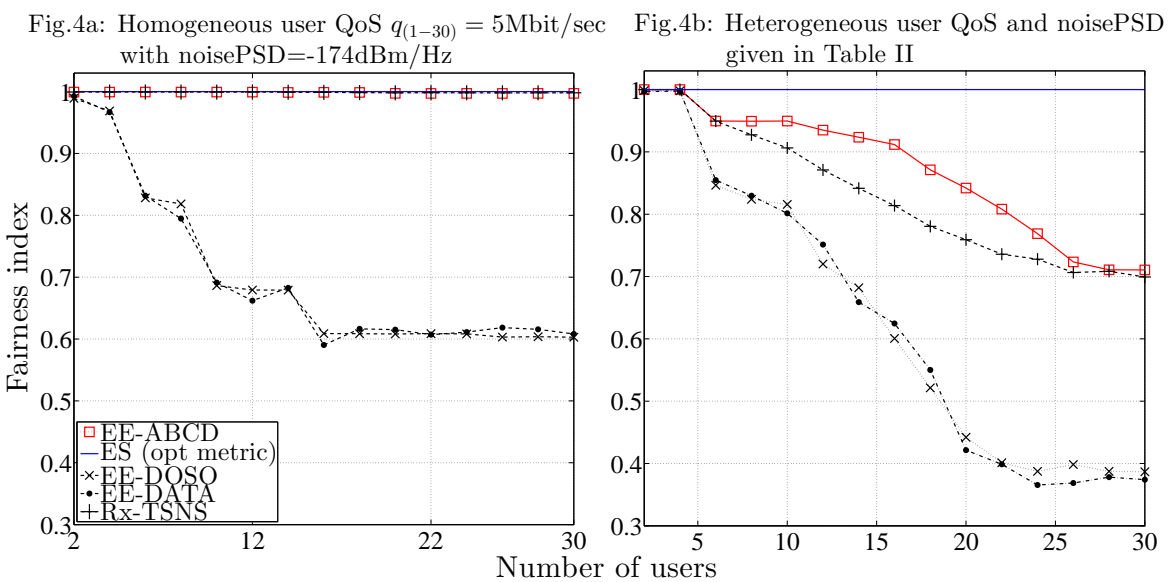

Fig. 4: Fairness index vs. number of users in homogeneous and heterogeneous large scale system: comparisons on fairness provision between the proposed EE-ABCD and relevant EE-DOSO, EE-DATA and Rx-TSNS 
TABLE II: User characteristics for simulations settings in Fig.4b and Fig.5

\begin{tabular}{ccccccccccc} 
user & $\mathbf{1}$ & 2 & 3 & 4 & 5 & $\mathbf{6}$ & 7 & 8 & 9 & 10 \\
$q_{k}(\mathrm{Mbit} / \mathrm{sec})$ & $\mathbf{5}$ & 5 & 5 & 5 & 5 & $\mathbf{8}$ & 5 & 5 & 5 & 5 \\
NoisePSD $(\mathrm{dBm} / \mathrm{Hz})$ & $\mathbf{- 1 2 7}$ & -174 & -174 & -174 & -174 & $\mathbf{- 1 0 2}$ & -174 & -174 & -174 & -174 \\
\hline user & 11 & $\mathbf{1 2}$ & 13 & 14 & 15 & 16 & 17 & $\mathbf{1 8}$ & 19 & 20 \\
$q_{k}(\mathrm{Mbit} / \mathrm{sec})$ & 5 & $\mathbf{7}$ & 5 & 5 & 5 & 5 & 5 & $\mathbf{6}$ & 5 & 5 \\
NoisePSD $(\mathrm{dBm} / \mathrm{Hz})$ & -174 & $\mathbf{- 1 8 2}$ & -174 & -174 & -174 & -174 & -174 & $\mathbf{- 1 8 2}$ & -174 & -174 \\
\hline user & 21 & 22 & 23 & 24 & 25 & 26 & 27 & 28 & 29 & 30 \\
$q_{k}(\mathrm{Mbit} / \mathrm{sec})$ & 5 & 5 & 5 & 5 & 5 & 5 & 5 & 5 & 5 & 5 \\
NoisePSD $(\mathrm{dBm} / \mathrm{Hz})$ & -174 & -174 & -174 & -174 & -174 & -174 & -174 & -174 & -174 & -174
\end{tabular}

(ii) all users suffer the same QoS loss under resource scarcity ${ }^{6}$.

More precisely, we set the resource sufficiency state by considering in Fig.4a that all channels have same SNR (noisePSD $=-174 \mathrm{dBm} / \mathrm{Hz}$ ) and all users request homogeneous QoS $\left(q_{\{1-30\}}=5 \mathrm{Mbit} / \mathrm{sec}\right.$, noisePSD $\left.=-174 \mathrm{dBm} / \mathrm{Hz}\right)$ such that the available power $p_{\max }$ at the $\mathrm{BS}$ is enough to serve all users. We see the proposed EE-ABCD along with the game theoretical Rx-TSNS are able to allocate the resources almost perfectly fair (i.e. all users are allocated with same throughputs) achieving similar FI to ES. On the other hand, EE-DOSO and EE-DATA score much lower FI $\rightarrow 0.62$ by opportunistically allocating more resources to some users meaning that some other users may not be able to satisfy their minimum QoS requirements [26].

Furthermore, we set resource scarcity, by considering in Fig.4b that the two users $k=\{1,6\}$ have heterogeneous QoS $q_{\{1,6\}}=\{5,8\}$ and suffer from noisy channels with noisePSD $=\{-127,-102,\} \mathrm{dBm} / \mathrm{Hz}$, while users $k=\{12,18\}$ with $q_{\{12,18\}}=\{7,6\}$ have best channel conditions among others with noisePSD $=\{-182,-182,\} \mathrm{dBm} / \mathrm{Hz}$, respectively, shown in Table II. As expected, the FIs of all schemes decrease with the increased number of users that join the system since the supplied to the BS power $p_{\max }$ is (on purpose) not enough. However, the EE-ABCD and Rx-TSNS score notably higher $F I$ s than EE-DOSO and EE-DATA schedulers, which affirms the practical difference between game-theoretical and opportunistic scheduling. That is, taking no consideration of fairness patterns practically leads to violation of the QoS

\footnotetext{
${ }^{6}$ Resource scarcity can be identified by observing the performance of each scheme at the boundary point beyond which the supplied power at the BS is not enough to accommodate all the users, and captures how fairly resources are allocated to users.
}

constraint (1) for some users even if the BS is supplied with enough power to support all users' minimum QoS (as also pointed out in Fig.2). For example, by examining in Fig.5c and Fig.5d the corresponding throughputs allocated by EEDOSO and EE-DATA to each individual user, respectively, we see that in both resource sufficiency and resource scarcity states, the minimum QoS of user $k=\{18\}$ (with best channel quality) is fulfilled within the large region $0 \leq K \leq 25$, while the QoS of user $k=\{1\}$ (with worst channels) within the much smaller region of $0 \leq K \leq 13$. More importantly, in Fig.4b the proposed EE-ABCD scores higher average $F I=0.91$ than the $F I=0.82$ of Rx-TSBS, which reveals the key difference between asymmetric and symmetric fair scheduling.

That is, in Blotto game strategies, users compete with each other to gain as many resources as possible, while in proportional NBS-based games users negotiate towards compromised (bargaining) solutions. This key difference between "competing" and "negotiating" seems to have significant impact on the behaviour of networks with increased degree of user and channel heterogeneity. For example, by observing Fig.5a and Fig.5b we see that (i) EE-ABCD satisfies all user's minimum QoS within region $1 \leq K \leq 23$, which is larger than the region $1 \leq K \leq 21$ of Rx-TSNS, and (ii) in resource scarcity state, Rx-TSNS throughputs decay at the same pace, while in EE$\mathrm{ABCD}$ the throughputs of less demanded users $k=\{1,18\}$ have larger decaying rate than throughputs of most demanded users $k=\{6,12\}$. In conclusion, Fig.4 and Fig.5 show that the proposed EE-ABCD provides higher throughputs than all other examined schemes by distributing radio resources more fairly in both homogeneous and heterogeneous system setting.

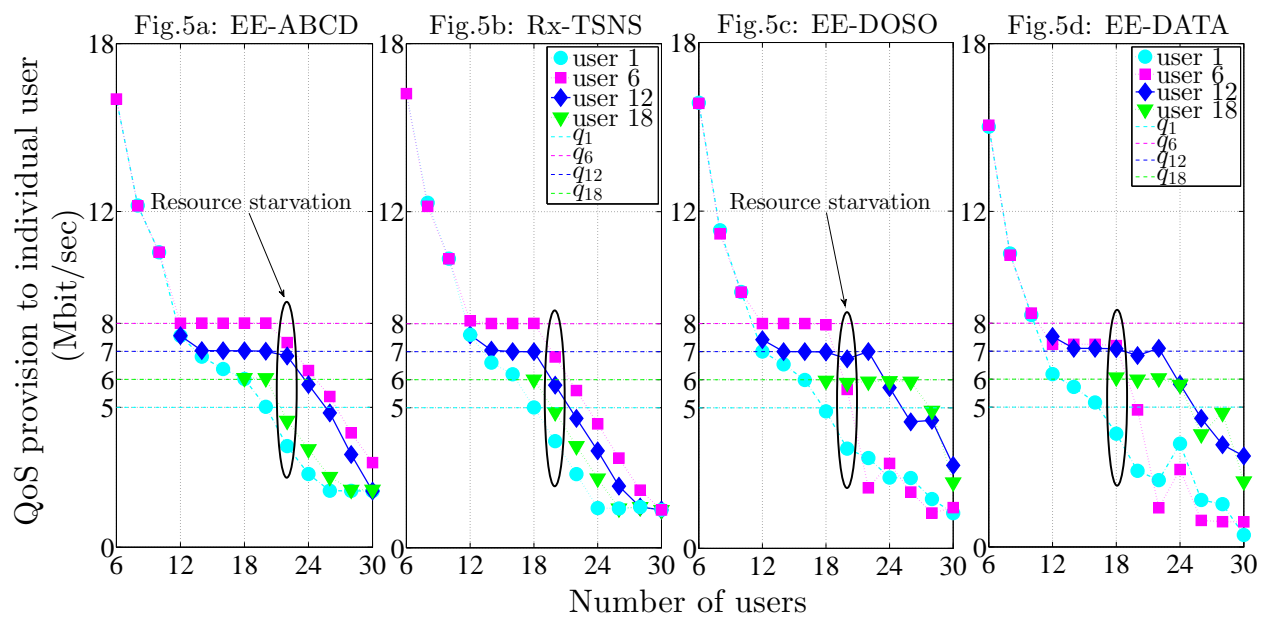

Fig. 5: QoS provision to individual users versus number of users. Comparisons on QoS performance between the proposed EE-ABCD and relevant EE-DOSO, EE-DATA and Rx-TSNS 


\section{CONCLUSION}

This paper developed a new sum- $E E$ maximisation scheme to derive joint optimal bandwidth and transmit power allocations using auction game theory. Our focus was drawn on a stochastic asymmetric Blotto game designed by considering the "competition of the majority", where multiple user utilities can be interdependent, which in turn, enables the resources to be fairly distributed among users. We show that with such feature our system can better capture the user heterogeneity, and improve fairness and performance compared to proportional games. In addition, we proposed a new solution method based on CCT and standard Lagrangian analysis, which renders the intermediate CCT coefficients and Lagrangian multipliers than commonly burden the optimisation analysis. This minimises the complexity of our solution so that the optimal points can be derived by solving a single-variable linear function for each user. We provided proofs with full analysis of key features and properties of our proposal in terms of the existence and uniqueness of NE, interdependency between users, Pareto boundaries, global optimality, implementation complexity and convergence. Finally, we provided simulation comparisons with relevant decision making systems to highlight the superiority of our scheme in terms of higher sum- $E E$ performance in significantly less time and with the guaranteed convergence. Our approach has the potential to be developed into generic slicing framework to accommodate next-generation applications under joint eMBB, mMTC, URLLC use cases.

\section{APPENDIX A}

PROOF OF THE OPTIMAL BANDWIDTH ALLOCATION SOLUTION

$$
\text { (7) IN THEOREM } 1
$$

Considering (B1) and (C1), as well as the definitions of $r_{k}^{n}$ and $u_{k}\left(p_{k}^{n}, b_{k}^{n}\right)$ in (1) and (5), respectively, we can write the Lagrangian function $L^{(1)}$ of problem (5) as

$$
\begin{array}{r}
L^{(1)}=\sum_{n=1}^{N}\left(\frac{b_{k}^{n}}{\sum_{l=1}^{N} b_{k}^{l}} \cdot w^{n} \cdot \log _{2}\left(1+\frac{p_{k}^{n} \cdot\left|h_{k}^{n}\right|^{2}}{b_{k}^{n} \cdot N_{0}}\right)\right) \\
-\nu^{n}\left(\sum_{k=1}^{K} b_{k}^{n}-w^{n}\right), \forall k,
\end{array}
$$

with $\nu^{n}$ the Lagrangian multiplier related to bandwidth constraint (C1). From the KKT conditions [31], the differentiation of $L$ w.t.r $b_{k}^{n}$ yields

$$
\begin{aligned}
& \left.\frac{\partial L}{\partial b_{k}^{n}}\right|_{\left\{p_{k}^{n}, b_{k}^{n}, \nu^{n}, S N R_{k}^{n}\right\}=\left\{p_{k}^{n *}, b_{k}^{n *}, \nu^{n *}, S N R_{k}^{n *}\right\}}=0 \Rightarrow \\
& \frac{\sum_{n^{\prime}=1, n^{\prime} \neq n}^{N} b_{k}^{n^{\prime} *}}{\left(\sum_{n^{\prime \prime}=1}^{N} b_{k}^{n^{\prime \prime} *}\right)^{2}} \cdot \frac{1}{\ln 2} \cdot\left[\ln \left(1+S N R_{k}^{n *}\right)-\frac{S N R_{k}^{n *}}{\left(1+S N R_{k}^{n *}\right)}\right. \\
& \left.\times\left(1+\frac{b_{k}^{n *}}{\sum_{n^{\prime}=1, n^{\prime} \neq n}^{N} b_{k}^{n^{\prime} *}}\right)\right] \Rightarrow \frac{\sum_{n^{\prime}=1, n^{\prime} \neq n}^{N} b_{k}^{n^{\prime} *}}{\left(\sum_{n^{\prime \prime}=1}^{N} b_{k}^{n^{\prime \prime} *}\right)^{2}} \cdot r_{k}^{n *}=\frac{\nu^{n *}}{w^{n}},
\end{aligned}
$$

where $r_{k}^{n *}=\frac{1}{\ln 2} \cdot\left[\ln \left(1+S N R_{k}^{n *}\right)-\frac{S N R_{k}^{n *}}{\left(1+S N R_{k}^{n *}\right)}\right.$ $\left.\times\left(1+\frac{b_{k}^{n *}}{\sum_{n^{\prime}=1, n^{\prime} \neq n}^{N} b_{k}^{n^{\prime *}}}\right)\right]$. We rewrite relation (17) as

$$
\begin{gathered}
\frac{\sum_{n^{\prime}=1, n^{\prime} \neq n}^{N} b_{k}^{n^{\prime} *}}{\left(\sum_{n^{\prime \prime}=1}^{N} b_{k}^{n^{\prime \prime *}}\right)^{2}} \cdot r_{k}^{n *}=\ldots=\frac{\sum_{n^{\prime}=1, n^{\prime} \neq n}^{N} b_{k^{\prime}}^{n^{\prime} *}}{\left(\sum_{n^{\prime \prime}=1}^{N} b_{k^{\prime}}^{n^{\prime \prime} *}\right)^{2}} \cdot r_{k^{\prime}}^{n *}=\ldots \\
\ldots=\frac{\sum_{n^{\prime}=1, n^{\prime} \neq n}^{N} b_{K}^{n^{\prime} *}}{\left(\sum_{n^{\prime \prime}=1}^{N} b_{K}^{n^{\prime \prime} *}\right)^{2}} \cdot r_{K}^{n *}=\frac{\nu^{n *}}{w^{n}},
\end{gathered}
$$

which, recalling the budget $b_{k}=\sum_{n=1}^{N} b_{k}^{n^{*}}$, calculates to

$$
\begin{array}{r}
\frac{\sum_{n^{\prime}=1, n^{\prime} \neq n}^{N} b_{k}^{n^{\prime} *}}{\left(b_{k}\right)^{2}} \cdot r_{k}^{n *}=\ldots=\frac{\sum_{n^{\prime}=1, n^{\prime} \neq n}^{N} b_{k^{\prime}}^{n^{\prime} *}}{\left(b_{k^{\prime}}\right)^{2}} \cdot r_{k^{\prime}}^{n *}=\ldots \\
\ldots=\frac{\sum_{n^{\prime}=1, n^{\prime} \neq n}^{N} b_{K}^{n^{\prime} *}}{\left(b_{K}\right)^{2}} \cdot r_{K}^{n *}=\frac{\nu^{n *}}{w^{n}}, \Rightarrow
\end{array}
$$

$$
\begin{aligned}
r_{k}^{n *} \cdot\left(b_{k^{\prime}}\right)^{2} \cdot\left(\sum_{n^{\prime}=1, n^{\prime} \neq n}^{N} b_{k}^{n^{\prime} *}\right) & \\
& =r_{k^{\prime}}^{n *} \cdot\left(b_{k}\right)^{2} \cdot\left(\sum_{n^{\prime}=1, n^{\prime} \neq n}^{N} b_{k^{\prime}}^{n^{\prime} *}\right)
\end{aligned}
$$

By setting $B=\sum_{k=1}^{K} b_{k}=\sum_{k=1}^{K} \sum_{n=1}^{N} b_{k}^{n *}=\sum_{n=1}^{N} \sum_{k=1}^{K} b_{k}^{n *}=$ $\sum_{n=1}^{N} b^{n}$, relation (19) yields

$$
\begin{gathered}
b_{k^{\prime}}=b_{k} \cdot \frac{\sqrt{r_{k^{\prime}}^{n *} \cdot \sum_{n^{\prime \prime}=1, n^{\prime \prime} \neq n}^{N} b_{k^{\prime}}^{n^{\prime \prime *}}}}{\sqrt{r_{k}^{n *} \cdot \sum_{n^{\prime \prime}=1, n^{\prime \prime \neq} \neq n}^{N} b_{k}^{n^{\prime \prime *}}}} \Rightarrow \\
B=\sum_{k^{\prime}=1}^{K} b_{k^{\prime}}=b_{k} \cdot \frac{\sum_{k^{\prime}=1}^{K} \sqrt{r_{k^{\prime}}^{n *} \cdot \sum_{n^{\prime \prime}=1, n^{\prime \prime} \neq n}^{N} b_{k^{\prime}}^{n^{\prime \prime} *}}}{\sqrt{r_{k}^{n *} \cdot \sum_{n^{\prime \prime}=1, n^{\prime \prime \neq n}}^{N} b_{k}^{n^{\prime \prime *}}}} \Rightarrow \\
b_{k}=B \cdot \frac{\sqrt{r_{k}^{n *} \cdot \sum_{n^{\prime \prime}=1, n^{\prime \prime \neq n}}^{N} b_{k}^{n^{\prime \prime *}}}}{\sum_{k^{\prime}=1}^{K} \sqrt{r_{k^{\prime}}^{n *} \cdot \sum_{n^{\prime \prime}=1, n^{\prime \prime \neq n}}^{N} b_{k^{\prime}}^{n^{\prime \prime *}}}} .
\end{gathered}
$$

Further, with (20)-(22), relation (18) defines the ratio for carrier $n$ over carrier $n^{\prime}$ of user $k$ as

$$
\begin{array}{r}
\frac{r_{k}^{n *} \cdot \sum_{n^{\prime \prime}=1, n^{\prime \prime} \neq n}^{N} b_{k}^{n^{\prime \prime} *}}{r_{k}^{n^{\prime} *} \cdot \sum_{n^{\prime \prime}=1, n^{\prime \prime} \neq n^{\prime}}^{N} b_{k}^{n^{\prime \prime} *}}=\ldots=\frac{r_{k^{\prime}}^{n *} \cdot \sum_{n^{\prime \prime}=1, n^{\prime \prime} \neq n}^{N} b_{k^{\prime}}^{n^{\prime \prime} *}}{r_{k^{\prime}}^{n^{\prime} *} \cdot \sum_{n^{\prime \prime}=1, n^{\prime \prime} \neq n^{\prime}}^{N} b_{k^{\prime}}^{n^{\prime \prime} *}}=\ldots \\
\ldots=\frac{r_{K}^{n *} \cdot \sum_{n^{\prime \prime}=1, n^{\prime \prime} \neq n}^{N} b_{K}^{n^{\prime \prime}}}{r_{K}^{n^{\prime} *} \cdot \sum_{n^{\prime \prime}=1, n^{\prime \prime} \neq n^{\prime}}^{N} b_{K}^{n^{\prime \prime} *}}=\frac{\nu^{n *}}{\nu^{n^{\prime} *}} \Rightarrow \\
\frac{r_{k}^{n *} \cdot\left(b_{k}-b_{k}^{n *}\right)}{r_{k}^{n^{\prime} *} \cdot\left(b_{k}-b_{k}^{n^{\prime} *}\right)}=\ldots=\frac{r_{k^{\prime}}^{n *} \cdot\left(b_{k^{\prime}}-b_{k^{\prime}}^{n *}\right)}{r_{n^{\prime} *}^{n^{\prime} *}\left(b_{k^{\prime}}-b_{n^{\prime} *}^{n^{\prime}}\right)}=\ldots \\
\ldots=\frac{r_{K}^{n *} \cdot\left(b_{K}-b_{K}^{n *}\right)}{r_{K}^{n^{\prime} *} \cdot\left(b_{K}-b_{K}^{n^{\prime} *}\right)}=\frac{\nu^{n *}}{\nu^{n^{\prime} *}} .
\end{array}
$$

Relation (23) resolves to

$$
\begin{aligned}
& \nu^{n^{\prime} *} \cdot r_{k}^{n *} \cdot \sum_{n^{\prime \prime}=1, n^{\prime \prime} \neq n}^{N} b_{k}^{n^{\prime \prime} *}=\nu^{n *} \cdot r_{k}^{n^{\prime} *} \\
& \times \sum_{n^{\prime \prime}=1, n^{\prime \prime} \neq n^{\prime}}^{N} b_{k}^{n^{\prime \prime} *} \Rightarrow \frac{\nu^{n *}}{\nu^{n^{\prime *}}}=\frac{r_{k}^{n *} \cdot \sum_{n^{\prime \prime}}^{N}}{r_{k}^{n^{\prime} * 1, n^{\prime \prime} * n} \sum_{n^{\prime \prime}=1, n^{\prime \prime} \neq n^{\prime}}^{b^{n^{\prime \prime}}} b^{n^{\prime \prime} *}} \\
& =\frac{r_{k}^{n *} \cdot\left(B-b^{n *}\right)}{r_{n^{\prime}}^{*} \cdot\left(B-b^{n^{*} *}\right)} \text {. }
\end{aligned}
$$

By substituting (25) into (24) we get $\frac{\left(b_{k}-b_{k}^{n *}\right)}{\left(b_{k}-b_{k}^{n^{\prime *}}\right)}=\frac{\left(B-b^{n *}\right)}{\left(B-b^{n^{\prime *}}\right)}$, which can be reformed as

$$
\begin{aligned}
b_{k}^{n *} & =b_{k}-\frac{\left(B-b^{n *}\right)}{\left(B-b^{n^{\prime} *}\right)} \cdot\left(b_{k}-b_{k}^{n^{\prime} *}\right) \\
& =b_{k} \cdot \frac{\left(b^{n *}-b^{n^{\prime} *}\right)}{\left(B-b^{n^{\prime *}}\right)}+\frac{\left(B-b^{n *}\right)}{\left(B-b^{n^{\prime}}\right)} \cdot b_{k}^{n^{\prime *}} .
\end{aligned}
$$

By setting $b_{k}=\sum_{n=1}^{N} b_{k}^{n^{*}}$, relation (26) calculates to

$$
\begin{aligned}
b_{k}=b_{k} \cdot \sum_{n=1}^{N} \frac{\left(b^{n *}-b^{n^{\prime} *}\right)}{\left(B-b^{n^{\prime} *}\right)}+\sum_{n=1}^{N} \frac{\left(B-b^{n *}\right)}{\left(B-b^{n^{\prime} *}\right)} \cdot b_{k}^{n^{\prime} *} \\
=\frac{1}{\left(B-b^{n^{\prime} *}\right)}\left(b_{k} \cdot \sum_{n=1}^{N}\left(b^{n *}-b^{n^{\prime} *}\right)+B \cdot b_{k}^{n^{\prime} *} \cdot(N-1)\right) \Rightarrow \\
b_{k}^{n^{\prime} *}=\frac{b_{k} \cdot\left(B-b^{n^{\prime} *}\right)-b_{k} \cdot \sum_{n=1}^{N}\left(b^{n *}-b^{n^{\prime} *}\right)}{b_{k} \cdot\left(B-b^{n^{\prime} *}-B_{\dot{B}}(N \cdot)^{1 n^{\prime} *}\right)} \\
=\frac{B \cdot(N-1)}{B} \\
=b^{n^{\prime} *} \cdot \frac{b_{k}}{B} .
\end{aligned}
$$

By substituting (21) into (27) we obtain the optimal expression of $b_{k}^{n *}$ in (7) of Theorem 1. Note that due to constraint (C1), $b_{k}^{n *}$ is maximal for $b^{n}=w^{n}$. Also, due to the square root terms included in (7), $r_{k}^{n *}$ and $r_{k^{\prime}}^{n *}$ should be positive, otherwise $b_{k}^{n *}$ is a complex number.

Lemma 1. $r_{k}^{n *}$ in (7) is positive for $\beta_{k}^{n}=\frac{b_{k}^{n *}}{\sum_{n^{\prime}=1}^{N} b_{k}^{n^{\prime \prime}}}>0$.

Proof. Recall relation (17) to rewrite term $r_{k}^{n *}$ in (7) as

$$
\begin{aligned}
r_{k}^{n *} & =\ln \left(1+S N R_{k}^{n *}\right)-\frac{S N R_{k}^{n *}}{\left(1+S N R_{k}^{n *}\right)} \cdot\left(1+\frac{b_{k}^{n *}}{\sum_{n^{\prime}=1}^{N} b_{k}^{n^{\prime} *}}\right) \\
& =\ln \left(1+S N R_{k}^{n *}\right)-\frac{S N R_{k}^{n *}}{\left(1+S N R_{k}^{n *}\right)} \cdot\left(1+\beta_{k}^{n}\right) .
\end{aligned}
$$

Supposing $r_{k}^{n *}>0$, relation (28) yields

$$
\begin{gathered}
\left(1+S N R_{k}^{n *}\right) \cdot e^{-\left(1+\beta_{k}^{n}\right)} \cdot \ln \left(\left(1+S N R_{k}^{n *}\right) \cdot e^{-\left(1+\beta_{k}^{n}\right)}\right) \\
>-\left(1+\beta_{k}^{n}\right) \cdot e^{-\left(1+\beta_{k}^{n}\right)} .
\end{gathered}
$$


If $z=\ln \left(\left(1+S N R_{k}^{n *}\right) \cdot e^{-\left(1+\beta_{k}^{n}\right)}\right)$ then from Lambert-W function $W(\cdot)$ [36], (29) we resolve

$$
\begin{aligned}
& e^{z} \cdot z>-\left(1+\beta_{k}^{n}\right) e^{-\left(1+\beta_{k}^{n}\right)} \Rightarrow \\
& \left(1+S N R_{k}^{n *}\right)>\underbrace{e^{W\left(-\left(1+\beta_{k}^{n}\right) \cdot e^{-\left(1+\beta_{k}^{n}\right)}\right)+\left(1+\beta_{k}^{n}\right)} .}_{T_{1}} .
\end{aligned}
$$

Since $1+S N R_{k}^{n *}$ is real, $T_{1}$ in (30) is real too. Clearly, $T_{1}$ is real for $\beta_{k}^{n}>0$. This completes the proof of Lemma 1 and optimal bandwidth allocation (7) in Theorem 1.

\section{APPENDIX B}

PROOF OF THE OPTIMAL TRANSMIT POWER ALLOCATION SOLUTION (8) IN THEOREM 1

Considering (B2) and (C2)-(C4), we write the Lagrangian function $L^{(2)}$ of problem (6) as

$$
\begin{aligned}
L^{(2)} & =t \cdot \sum_{k=1}^{K} \sum_{n=1}^{N} \eta_{0} \cdot b_{k}^{n} \cdot \log _{2}\left(1+\frac{y_{k}^{n} \cdot\left|h_{k}^{n}\right|^{2}}{t \cdot b_{k}^{n} \cdot N_{0}}\right) \\
& +t \cdot\left(\sum_{k=1}^{K} \xi_{k} \cdot\left(\sum_{n=1}^{N} b_{k}^{n} \cdot \log _{2}\left(1+\frac{y_{k}^{n} \cdot\left|h_{k}^{n}\right|^{2}}{t \cdot b_{k}^{n} \cdot N_{0}}\right)-q_{k}\right)\right) \\
& +\mu \cdot\left(\sum_{k=1}^{K} \sum_{n=1}^{N}\left(\zeta \cdot y_{k}^{n}\right)+t \cdot p_{c}-1\right) \\
& -\lambda \cdot\left(\sum_{k=1}^{K} \sum_{n=1}^{N}\left(y_{k}^{n}\right)-t \cdot p_{\max }\right),
\end{aligned}
$$

where $\xi_{k}, \lambda$ and $\mu$ are the Lagrangian multipliers associated with constraints (C2), (C3) and (C4), respectively. According to the KKT conditions [31], the differentiation of $L^{(2)}$ in (21) w.r.t the CCT coefficient $t$ yields

$$
\begin{aligned}
& \left.\frac{\partial L}{\partial t}\right|_{\left\{p_{k}^{n}, y_{k}^{n}, t, \xi_{k}, \lambda, \mu\right\}=\left\{p_{k}^{n *}, y_{k}^{n *}, t^{*}, \xi_{k}^{*}, \lambda^{*}, \mu^{*}\right\}}=0 \Rightarrow \\
& \eta_{0} \cdot \sum_{k=1}^{K} \sum_{n=1}^{N} b_{k}^{n} \cdot \log _{2}\left(1+\frac{p_{k}^{n} \cdot\left|h_{k}^{n}\right|^{2}}{b_{k}^{n} \cdot N_{0}}\right) \\
& +\sum_{k=1}^{K} \xi_{k} \cdot\left(\sum_{n=1}^{N} b_{k}^{n} \cdot \log _{2}\left(1+\frac{p_{k}^{n} \cdot\left|h_{k}^{n}\right|^{2}}{b_{k}^{n} \cdot N_{0}}\right)-q_{k}\right) \\
& -\left(\sum_{k=1}^{K} \sum_{n=1}^{N} \frac{\left(\eta_{0}+\xi_{k}\right) \cdot\left|h_{k}^{n}\right|^{2}}{\ln 2 \cdot N_{0} \cdot\left(1+\frac{\left.p_{k}^{n} \cdot||_{k}^{n}\right|^{2}}{b_{k}^{n} \cdot N_{0}}\right)}\right) \\
& +\mu \cdot p_{c}+\lambda \cdot p_{\max }=0 .
\end{aligned}
$$

Also, the differentiation of $L^{(2)}$ in (31) w.r.t the CCT coefficient $y_{k}^{n}$ yields

$$
\left.\frac{\partial L}{\partial y_{k}^{n}}\right|_{\left\{p_{k}^{n}, y_{k}^{n}, t, \xi_{k}, \lambda, \mu\right\}=\left\{p_{k}^{n *}, y_{k}^{n *}, t^{*}, \xi_{k}^{*}, \lambda^{*}, \mu^{*}\right\}}=0 \Rightarrow
$$

Multiplying (33) with $p_{k}^{n *}$ and summarising w.r.t $k$ and $n$ we have

$$
\begin{aligned}
\sum_{k=1}^{K} \sum_{n=1}^{N} \frac{p_{k}^{n *} \cdot\left|h_{k}^{n}\right|^{2} \cdot\left(\eta_{0}+\xi_{k}^{*}\right)}{\ln 2 \cdot N_{0} \cdot\left(1+\frac{p_{k}^{n *} \cdot\left|h_{k}^{n}\right|^{2}}{b_{k}^{n *} \cdot N_{0}}\right)} \\
\quad=\left(\lambda^{*}-\zeta \cdot \mu^{*}\right) \cdot \sum_{k=1}^{K} \sum_{n=1}^{N}\left(p_{k}^{n *}\right),
\end{aligned}
$$

which can be substituted into (32) to derive

$$
\begin{aligned}
& \frac{\eta_{0} \cdot \sum_{k=1}^{K} \sum_{n=1}^{N} b_{k}^{n *} \cdot \log _{2}\left(1+\frac{p_{k}^{n *} \cdot\left|h_{k}^{n}\right|^{2}}{b_{k}^{n *} \cdot N_{0}}\right)}{\left(\sum_{k=1}^{K} \sum^{N}\left(\zeta \cdot p_{k}^{n *}\right)+p_{c}\right)} \\
& \left(\sum_{k=1}^{K} \sum_{n=1}^{N}\left(\zeta \cdot p_{k}^{n *}\right)+p_{c}\right) \\
& +\frac{\sum_{k=1}^{K} \xi_{k} \cdot\left(\sum_{n=1}^{N} b_{k}^{n *} \cdot \log _{2}\left(1+\frac{p_{k}^{n *} \cdot\left|h_{k}^{n}\right|^{2}}{b_{k}^{n *} \cdot N_{0}}\right)-q_{k}\right)}{\left(\sum_{k=1}^{K} \sum_{n=1}^{N}\left(\zeta \cdot p_{k}^{n *}\right)+p_{c}\right)} \\
& +\lambda^{*} \cdot \frac{\left(p_{\max }-\sum_{k=1}^{K} \sum_{n=1}^{N} p_{k}^{n *}\right)}{\left(\sum_{k=1}^{K} \sum_{n=1}^{N}\left(\zeta \cdot p_{k}^{n *}\right)+p_{c}\right)}+\mu^{*}=0 .
\end{aligned}
$$

Recalling the KKT conditions of complementary slackness, i.e., $\lambda^{*}=0, \sum_{k=1}^{K} \sum_{n=1}^{N}\left(p_{k}^{n *}\right)-p_{\max }>0$ or $\lambda^{*}>0$, $\sum_{k=1}^{K} \sum_{n=1}^{N}\left(p_{k}^{n *}\right)-p_{\max }=0$ and $\xi_{k}=0, \sum_{n=1}^{N} b_{k}^{n *}$. $\log _{2}\left(1+\frac{p_{k}^{n *} \cdot\left|h_{k}^{n}\right|^{2}}{b_{k}^{n *} \cdot N_{0}}\right)-q_{k}>0$ or $\xi_{k}>0, \sum_{n=1}^{N} b_{k}^{n *}$. $\log _{2}\left(1+\frac{p_{k}^{n *} \cdot\left|h_{k}^{n}\right|^{2}}{b_{k}^{n *} \cdot N_{0}}\right)-q_{k}=0$, relation (35) becomes

$$
\frac{\sum_{k=1}^{K} \sum_{n=1}^{N} \eta_{0} \cdot b_{k}^{n *} \cdot \log _{2}\left(1+\frac{p_{k}^{n *} \cdot\left|h_{k}^{n}\right|^{2}}{b_{k}^{n *} \cdot N_{0}}\right)}{\sum_{k=1}^{K} \sum_{n=1}^{N}\left(\zeta \cdot p_{k}^{n *}\right)+p_{c}}+\mu^{*}=0,
$$

otherwise the available power at the BS $p_{\max }$ can never be totally exploited and/or the minimum user QoS requirement $q_{k}$ may not be always satisfied [31]. A conventional way to resolve relation (36) w.r.t the optimal $p_{k}^{n *} s$ is to calculate the user powers $K$-times per iteration considering that each user's QoS requirement $q_{k}$ is dependent on the (unknown) multiplier $\mu^{*}$, as shown in [21]-[30], [35]. Instead, we shall correlate the $Q o S$ requirements between users such that $p_{k}^{n *} s$ can be independent from $\mu^{*}$ and thus, they can be calculated only once per iteration for all users, which significantly reduces complexity. Particularly, from the CCT-oriented relation (33) we observe that for each user $k$ there exists a common coefficient $\omega_{k}$ for all carriers, i.e.,

$$
\begin{aligned}
\omega_{k}:=\omega_{k}^{n} & =\ldots=\omega_{k}^{m}=\frac{1+\frac{p_{k}^{n *} \cdot\left|h_{k}^{n}\right|^{2}}{b_{k}^{n *} \cdot N_{0}}}{\left|h_{k}^{n}\right|^{2} / N_{0}}=\ldots \\
\ldots & =\frac{1+\frac{p_{k}^{m *} \cdot\left|h_{k}^{m}\right|^{2}}{b_{k}^{m *} \cdot N_{0}}}{\left|h_{k}^{m}\right|^{2} / N_{0}}=\frac{\eta_{0}+\xi_{k}^{*}}{\ln 2 \cdot\left(\lambda^{*}-\mu^{*} \cdot \zeta\right)}, \forall n \neq m,
\end{aligned}
$$

which describes the optimal transmit power operand as

$$
p_{k}^{n *}=b_{k}^{n *} \cdot\left(\omega_{k}-\frac{N_{0}}{\left|h_{k}^{n}\right|^{2}}\right) \text {. }
$$

In addition, recalling our definition of user's throughput in (1) and by using (37), we calculate the QoS ratio $\gamma_{k}=\frac{q_{k}}{q_{k^{\prime}}}$ between the $k$-th and $k^{\prime}$-th user over all $N$ available carrier as

$$
\begin{aligned}
& \gamma_{k}=\frac{q_{k}}{q_{k^{\prime}}}=\frac{\sum_{n=1}^{N} b_{k}^{n *} \cdot \log _{2}\left(1+\frac{p_{k}^{n *}\left|h_{k}^{n}\right|^{2}}{b_{k}^{n *} \cdot N_{0}}\right)}{\sum_{n=1}^{N} b_{k^{\prime}}^{n *} \cdot \log _{2}\left(1+\frac{p_{1}^{n *}\left|h_{k^{\prime}}^{n}\right|^{2}}{b_{k^{\prime}}^{n *} \cdot N_{0}}\right)}
\end{aligned}
$$

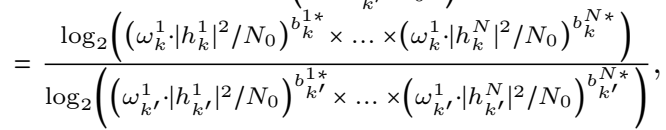

which with some manipulations can be resolved w.r.t coefficient $\omega_{k}$ as

$$
\omega_{k}=\omega_{k^{\prime}} \gamma_{k} \cdot \frac{b_{k^{\prime}}}{b_{k}} \cdot\left(\prod_{n=1}^{N}\left(\frac{\left|h_{k^{\prime}}^{n}\right|^{2}}{N_{0}}\right)^{b_{k^{\prime}}^{n *}}\right)^{\frac{\gamma_{k}}{b_{k}}} / \prod_{n=1}^{N}\left(\frac{\left|h_{k}^{n}\right|^{2}}{N_{0}}\right)^{\frac{b_{k}^{n *}}{b_{k}}}
$$

With relations (40) and (38) the total power consumption (denominator of (36)) is rewritten as

$$
\begin{aligned}
\sum_{k=1}^{K} \sum_{n=1}^{N} & p_{k}^{n *}=\sum_{k=1}^{K} \sum_{n=1}^{N} b_{k}^{n *} \cdot\left(\omega_{k}-\frac{N_{0}}{\left|h_{k}^{n}\right|^{2}}\right) \\
= & \sum_{k=1}^{K} \sum_{n=1}^{N} b_{k}^{n *} \cdot\left(\omega_{k^{\prime}} \frac{\gamma_{k^{\prime}} \cdot b_{k^{\prime}}}{b_{k}}\right. \\
& \left.\times \prod_{n=1}^{N}\left(\frac{\left(\left|h_{k^{\prime}}^{n}\right|^{2} / N_{0}\right)^{\gamma^{*} \cdot b^{n} b^{\prime \prime}}}{\left(\left|h_{k}^{m}\right|^{2} / N_{0}\right)^{b_{k}^{n *}}}\right)^{\frac{1}{b_{k}}}-\frac{N_{0}}{\left|h_{k}^{n}\right|^{2}}\right),
\end{aligned}
$$

while the total throughput (numerator of (36)) is rewritten as

$$
\begin{aligned}
& \eta_{0} \cdot \sum_{k=1}^{K} \sum_{n=1}^{N} b_{k}^{n *} \cdot \log _{2}\left(1+\frac{p_{k}^{n *} \cdot\left|h_{k}^{n}\right|^{2}}{b_{k}^{n *} \cdot N_{0}}\right)= \\
& \eta_{0} \cdot\left(\sum_{k=1}^{K} \gamma_{k}\right) \cdot \log _{2}\left(\left(\omega_{k^{\prime}}\right)^{b_{k^{\prime}}} \cdot \prod_{n=1}^{N}\left(\left|h_{k}^{n}\right|^{2} / N_{0}\right)^{b_{k^{\prime}}^{n *}}\right) .
\end{aligned}
$$

By substituting the power and throughput formulas (41) and (42), respectively, into (36), we get

$$
\begin{aligned}
& \eta_{0} \cdot\left(\sum_{k=1}^{K} \gamma_{k}\right) \cdot \log _{2}\left(\left(\omega_{k^{\prime}}\right)^{b} k^{\prime} \cdot \prod_{n=1}^{N}\left(\frac{\mid h_{k^{\prime}}^{n} \prime^{2}}{N_{0}}\right)^{b_{k^{\prime}}^{n *}}\right)
\end{aligned}
$$

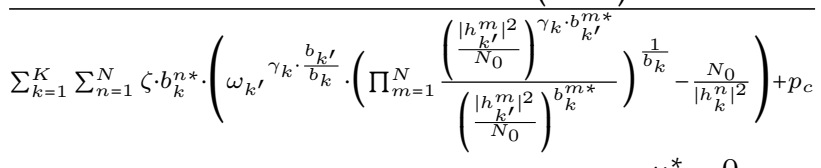

$$
\begin{aligned}
& -\mu^{*}=0 \text {, }
\end{aligned}
$$


The differentiation of (43) w.r.t $\omega_{k^{\prime}}$ yields a relation over the optimal coefficient $\omega_{k^{\prime}}^{*}$, i.e.,

$$
\begin{aligned}
& \sum_{k=1}^{K} \sum_{n=1}^{N} \zeta \cdot b_{k}^{n *} \cdot\left(\left(\omega_{k^{\prime}}^{*}\right)^{\gamma_{k} \cdot \frac{b_{k^{\prime}}}{b_{k}}} \cdot\left(\prod_{m=1}^{N} \frac{\left(\left|h_{k^{\prime}}^{m}\right|^{2}\right)^{\gamma_{k} \cdot b_{k^{\prime}}^{m *}}}{\left(\left|h_{k^{\prime}}^{m}\right|^{2}\right)^{b_{k^{\prime}}^{n *}}}\right)^{\frac{k^{\prime}}{b_{k}}}\right. \\
& \left.\times\left(1-\ln \left(\prod_{m=1}^{N}\left(\omega_{k^{\prime}}^{*} \cdot\left|h_{k^{\prime}}^{m}\right|^{2}\right)^{\gamma_{k} \cdot \frac{b_{k^{\prime}}^{m *}}{b_{k}}}\right)\right)-\frac{N_{0}}{\left|h_{k}^{n}\right|^{2}}\right)+p_{c}=0,
\end{aligned}
$$

which, by using Lambert-W function $W(\cdot)$ properties [36], resolves to

$$
\omega_{k^{\prime}}^{*}=\frac{\frac{p_{c}}{\zeta \cdot b_{k^{\prime}}^{m *} \cdot K \cdot N}-\frac{N_{0}}{\left|h_{k^{\prime}}^{m}\right|^{2}}}{W\left(\prod_{m=1}^{N}\left(\frac{\left|h_{k^{\prime}}^{m}\right|^{2}}{N_{0}}\right)^{\frac{b_{k^{\prime}}^{m *}}{b} k^{\prime}} \cdot\left(\frac{p_{c}}{\zeta \cdot b_{k^{\prime}}^{m *} \cdot K \cdot N}-\frac{N_{0}}{\left|h_{k^{\prime}}^{m}\right|^{2}}\right) \cdot e^{-1}\right)}
$$

The optimal coefficient $\omega_{k^{\prime}}^{*}$ in (45) can be easily computed as it is independent from multiplier $\mu^{*}$ and depends on known system variables only. Once we compute $\omega_{k^{\prime}}^{*}$, we can calculate $\omega_{k}^{*}$ from (40) (using one iteration for all the $K$ users) and substitute it into (38) to obtain the optimal powers $\left\{p_{k}^{n *}\right\}$. This completes the proof of the optimal transmit power (8) in Theorem 1.

\section{APPENDIX C
PROOF OF PROPOSITION 1}

To ensure non-singularity of the Jacobian matrix $J_{B_{k}^{*}}$ of function $B_{k}^{*}$ in (9) it is sufficient to show that its diagonal elements are equal to -1 and its off-diagonal elements equal to zero, i.e., $\frac{\partial B_{k}^{*}}{\partial b_{k}^{* *}}=-1$ and $\frac{\partial B_{k}^{*}}{\partial b_{k^{\prime}}^{n^{\prime *}}}=0$, respectively [35]. Let us set $\varphi_{b_{k}^{n *}}=\sqrt{r_{k}^{n *} \cdot \sum_{n^{\prime}=1, n^{\prime} \neq n}^{N} b_{k}^{n^{\prime *}}}$ and $\varphi_{-b_{k}^{n *}}=$ $\sum_{k^{\prime}=1, k^{\prime} \neq k}^{K} \sqrt{r_{k^{\prime}}^{n *} \cdot \sum_{n^{\prime}=1, n^{\prime} \neq n}^{N} b_{k^{\prime}}^{n^{\prime *}}}$ to express $B_{k}^{*}$ in (9) in simple form as

$$
\begin{aligned}
B_{k}^{*} & =-b_{k}^{n *}+b^{n} \cdot \frac{\sqrt{r_{k}^{n *} \cdot \sum_{n^{\prime}=1, n^{\prime} \neq n}^{N} b_{k}^{n^{\prime} *}}}{\sum_{k^{\prime}=1}^{K} \sqrt{r_{k^{\prime}}^{n *} \sum_{n^{\prime}=1, n^{\prime} \neq n}^{N} b_{k^{\prime}}^{n^{\prime} *}}} \\
& =\frac{\varphi_{b_{k}^{n *}}^{n *} b^{n}}{\varphi_{b_{k}^{n *}+\varphi_{-b_{k}^{n *}}}^{n *}}-b_{k}^{n *}=1-b_{k}^{n *}-\frac{\varphi_{-b_{k}^{n *} \cdot b^{n}}^{\left(\varphi_{b_{k}^{n *}}^{n *} \varphi_{-b_{k}^{n *}}\right)}}{} .
\end{aligned}
$$

The diagonal elements $\frac{\partial B_{k}^{*}}{\partial b_{k}^{* *}}$ of the Jacobian matrix $J_{B_{k}^{*}}$ of $B_{k}^{*}$ are calculated as

$$
\begin{aligned}
& \frac{\partial B_{k}^{*}}{\partial b_{k}^{n *}}=-1-\underbrace{\frac{1}{2 \cdot \ln 2} \cdot \frac{\varphi_{-b_{k}^{n *}}}{\left(\varphi_{b_{k}^{n *}}+\varphi_{-b_{k}^{n *}}\right)}}_{T_{2}} \\
& \times \underbrace{\frac{1}{\ln \left(1+\frac{p_{k}^{n *} \cdot\left|h_{k}^{n}\right|^{2}}{b_{k}^{n *} \cdot N_{0}}\right)-\frac{\frac{p_{k}^{n *}\left|h_{k}^{n}\right|^{2}}{b_{k}^{n *} \cdot N_{0}}}{1+\frac{p_{k}^{n *} \cdot\left|h_{k}^{n}\right|^{2}}{b_{k}^{n *} \cdot N_{0}}}-\beta_{k}^{n} \cdot \frac{\frac{p_{k}^{n *} \cdot\left|h_{k}^{n}\right|^{2}}{b_{k}^{n *} \cdot N_{0}}}{1+\frac{p_{k}^{n *} \cdot\left|h_{k}^{n}\right|^{2}}{b_{k}^{n *} \cdot N_{0}}}}}_{T_{3}}
\end{aligned}
$$

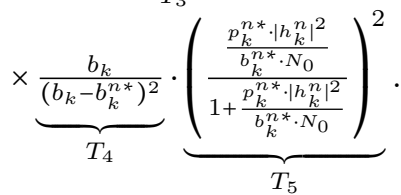

In order for $\frac{\partial B_{k}^{*}}{\partial b_{k}^{n *}}=-1$, terms $T_{2} \times T_{3} \times T_{4} \times T_{5}$ in (47) should be equal to zero. Since $T_{2}, T_{4}, T_{5}$ are less than one, we thus seek for a condition to ensure that term $T_{3}$ is less than one too, i.e.,

$$
\ln \left(1+\frac{p_{k}^{n *} \cdot\left|h_{k}^{n}\right|^{2}}{b_{k}^{n *} \cdot N_{0}}\right)-\frac{\frac{p_{k}^{n *} \cdot\left|h_{k}^{n}\right|^{2}}{b_{k}^{n *} \cdot N_{0}}}{1+\frac{p_{k}^{n *}\left|h_{k}^{n}\right|^{2}}{b_{k}^{n *} \cdot N_{0}}}-\beta_{k}^{n} \cdot \frac{\frac{p_{k}^{n *} \cdot\left|h_{k}^{n}\right|^{2}}{b_{k}^{n *} \cdot N_{0}}}{1+\frac{p_{k}^{n *}\left|h_{k}^{n}\right|^{2}}{b_{k}^{n *} \cdot N_{0}}}>1 .
$$

$$
\begin{aligned}
1+\frac{p_{k}^{n *} \cdot\left|h_{k}^{n}\right|^{2}}{b_{k}^{n *} \cdot N_{0}} & >e^{W\left(-\left(1+\beta_{k}^{n}\right) \cdot e^{-\left(2+\beta_{k}^{n}\right)}\right)+\left(2+\beta_{k}^{n}\right)} \Rightarrow \\
S N R_{k}^{n *} & >e^{W\left(-\left(1+\beta_{k}^{n}\right) \cdot e^{-\left(2+\beta_{k}^{n}\right)}\right)+\left(2+\beta_{k}^{n}\right)}-1 .
\end{aligned}
$$

$S N R_{k}^{n *}$ in (49) is real for $\beta_{k}^{n} \geq 1 e^{-4}$, which means that the diagonal elements of the Jacobian matrix $J_{B_{k}^{*}}$ are $\frac{\partial B_{k}^{*}}{\partial b_{k}^{n *}} \approx-1$ for $S N R_{k}^{n *}>5.306 \mathrm{~dB}$. Similarly, we have

$$
\begin{aligned}
\frac{\partial B_{k}^{*}}{\partial b_{k^{\prime}}^{n^{\prime} *}}=-\frac{1}{2} & \times \underbrace{\frac{1}{\ln \left(1+\frac{p_{k}^{n *} \cdot\left|h_{k}^{n}\right|^{2}}{b_{k}^{n *} \cdot N_{0}}\right)}}_{\Lambda_{1}} \times \underbrace{\frac{\frac{p_{k}^{n *} \cdot\left|h_{k}^{n}\right|^{2}}{b_{k}^{n *} \cdot N_{0}}}{\left(1+\frac{p_{k}^{n *} \cdot\left|h_{k}^{n}\right|^{2}}{b_{k}^{n *} \cdot N_{0}}\right)}}_{\Lambda_{3}} \\
& \times \underbrace{\frac{\varphi_{b_{k}^{n}}}{\left(\varphi_{\left.b_{k}^{n *}+\varphi_{-b_{k}^{n *}}\right)}\right.}}_{\varphi_{k}^{n *}}, k^{\prime} \in K, k^{\prime} \neq k .
\end{aligned}
$$

Terms $\Lambda_{1}, \Lambda_{2}$ and $\Lambda_{3}$ in (50) are positive thus, $\frac{\partial B_{k}^{*}}{\partial b_{k^{\prime}}^{n^{\prime *}}}<0$, which implies that the impact of the off-diagonal elements on the non-singularity of $J_{B_{k}^{*}}$ is negligible. Moreover, (47) confirms that the Jacobian matrix $J_{B_{k}^{*}}$ is continuous w.r.t $b_{k}^{n *}$, which certifies that $b_{k}^{n *}$ in (7) does exist. Similarly, we can show that $p_{k}^{n *}$ in (8) exists as well and thus, the existence of a NE in $\mathbf{B}$ is ensured for both $p_{k}^{n *}$ and $b_{k}^{n *}$. This completes the proof of Proposition 1.

APPENDIX D PROOF OF PROPOSITION 2

At time $t$, each best response function $\boldsymbol{f}\left(b_{k}^{n *}(t)\right)$ of set $\boldsymbol{f}\left(\mathbf{b}_{k}^{*}(t)\right)$ in (13) can be calculated as

$$
\begin{aligned}
& \boldsymbol{f}_{k}\left(b_{k}^{n *}(t)\right)=b_{k}^{n *}-b^{n} \cdot \frac{\sqrt{r_{k}^{n *} \cdot \sum_{n^{\prime}=1, n^{\prime} \neq n}^{N} b_{k}^{n^{\prime *}}}}{\sum_{k^{\prime}=1}^{K} \sqrt{r_{k^{\prime}}^{n *} \cdot \sum_{n^{\prime}=1, n^{\prime} \neq n}^{N} b_{k^{\prime}}^{n^{\prime} *}}} \\
& =b_{k}^{n *}-\frac{\sum_{k^{\prime}=1, k^{\prime}=k}^{K} \sqrt{r_{k^{\prime}}^{n *} \cdot \sum_{n^{\prime}=1, n^{\prime} \neq n}^{N} b^{n^{\prime} * *}}}{\sqrt{r_{k}^{n *} \cdot \sum_{n^{\prime}=1, n^{\prime} \neq n}^{N} b_{k}^{n^{\prime \prime}}}} \\
& =b_{k}^{n *}-\frac{b^{n}}{1+\frac{G}{\sqrt{\log _{2}\left(\left(1+\frac{p_{k}^{n *} \cdot\left|h_{k}^{n}\right|^{2}}{b_{k}^{n *} \cdot N_{0}}\right) e^{-1}\right)}}},
\end{aligned}
$$

where $G=\frac{\sum_{k^{\prime}=1, k^{\prime}=k}^{K} \sqrt{r_{k^{\prime}}^{n *} \cdot \sum_{n^{\prime}=1, n^{\prime} \neq n}^{N} b_{k^{\prime}}^{n^{\prime} *}}}{\sqrt{\sum_{n^{\prime}=1, n^{\prime} \neq n}^{N} b_{k^{\prime}}^{n^{\prime} *}}}>0$ has been added for brevity, $\bar{r}_{k}^{n *}=\log _{2}\left(\frac{1+\overline{S N R}_{k}^{n *}}{e}\right)$ is specified in (12). As observed, the right hand side of $\boldsymbol{f}\left(b_{k}^{n *}(t)\right)$ in (51) is monotone convex w.r.t $b_{k}^{n *}$ for a given $p_{k}^{n *}$, hence, recalling that $b_{k}^{n *} \in\left(0,8 \times 10^{6}\right]$, we calculate $\lim _{b_{k}^{n *} \rightarrow 0^{+}} f\left(b_{k}^{n *}\right)=0^{+}-b^{n}<0$, and $\lim _{b_{k}^{n *} \rightarrow+\infty} f\left(b_{k}^{n *}\right)=+\infty-0>0$. Therefore, according to the Intermediate Value Theorem [37], each $\boldsymbol{f}\left(b_{k}^{n *}(t)\right)$ of $\boldsymbol{f}\left(\mathbf{b}_{k}^{*}(t)\right)$ has at least one root in $\left(0,8 \times 10^{6}\right]$. Furthermore, to show that $\boldsymbol{f}\left(\mathbf{b}_{k}^{*}(t)\right)$ is comprised by standard functions it is sufficient to prove that it is jointly (i) positive, (ii) monotonous and (iii) scalable w.r.t the bandwidth allocation vector $\mathbf{b}_{k}^{*}(t)=\left(b_{k}^{1 *}, b_{k}^{2 *}, \ldots, b_{k}^{n *}, \ldots, b_{k}^{N *}\right)$ [38], [39].

(i) Positivity: Considering multiple users (i.e. $\left.k^{\prime} \neq k\right), \boldsymbol{f}\left(\mathbf{b}_{k}^{*}(t)\right)$ in (13) is positive because $r_{k}^{n *}>0, r_{k^{\prime}}^{n *}>0, \sum_{n^{\prime}=1, n^{\prime} \neq n}^{N} b_{k}^{n *}>0$ and $\sum_{n^{\prime}=1, n^{\prime} \neq n}^{N} b_{k^{\prime}}^{n^{\prime} *}>0$ for all $k, k^{\prime} \in K$.

(ii) Monotonicity: Considering multiple carriers $n \neq n^{\prime}$ and that user $k$ changes a bandwidth resource $b_{k}^{n *}$ by an amount of $0<\rho<1$, the bandwidth allocation vector of user $k$ becomes $\tilde{\mathbf{b}}_{k}^{*}(t+1)=\left(b_{k}^{1 *}, b_{k}^{2 *}, \ldots, \rho \cdot b_{k}^{n *}, \ldots, b_{k}^{N *}\right)$ and we can write the 


$$
\begin{aligned}
& \nabla g_{0}\left(y_{k}^{n}, t\right)=\left\{\begin{array}{l}
\frac{\partial g_{0}\left(y_{k}^{n}, t\right)}{\partial y_{k^{\prime}}^{n}}=1, \forall k, n \\
\frac{\partial g_{0}\left(y_{k}^{n}, t\right)}{\partial t}=-p_{\max }
\end{array}, \nabla g_{K+1}\left(y_{k}^{n}, t\right)=\left\{\begin{array}{l}
\frac{\partial g_{K+1}\left(y_{k}^{n}, t\right)}{\partial y_{k}^{n}}=\varsigma, \forall k \in K, n \in N \\
\frac{\partial g_{K+1}\left(y_{k}^{n}, t\right)}{t}=p_{c}
\end{array}\right.\right. \\
& \nabla g_{k}^{n}\left(y_{k}^{n}, t\right)=\left\{\begin{array}{c}
\frac{\partial g_{k}^{n}\left(y_{k}^{n}, t\right)}{\partial y_{k^{\prime}}^{n}}=\left\{\begin{array}{c}
\frac{\left|h_{k}^{n}\right|^{2}}{N_{0}} \cdot \frac{1}{\ln 2\left(1+S N R_{k}^{n}\right)}, \text { for } k^{\prime}=k, \forall k^{\prime}, k \in K \text { and } \forall n=N \\
0, \text { for } k^{\prime} \neq k
\end{array}\right. \\
\frac{\partial g_{k}^{n}\left(y_{k}^{n}, t\right)}{\partial t}=\sum_{n=1}^{N}\left(\frac{b_{k}^{n}}{\ln 2} \cdot\left(\ln \left(1+S N R_{k}^{n}\right)-\frac{S N R_{k}^{n}}{\left(1+S N R_{k}^{n}\right)}\right)\right)-q_{k}
\end{array}\right.
\end{aligned}
$$

derivation of its winning strategy as

$$
\begin{aligned}
& \boldsymbol{f}\left(\mathbf{b}_{k}^{*}(t)\right)-\boldsymbol{f}\left(\tilde{\mathbf{b}}_{k}^{*}(t)\right)=b^{n} \cdot \frac{\sqrt{r_{k}^{n *}\left(b_{k}^{n *}\right) \cdot \sum_{n^{\prime}=1, n^{\prime} \neq n}^{N} b_{k}^{n^{\prime} *}}}{\sqrt{\frac{v^{n *}}{w^{n}} \cdot B}} \\
&-\tilde{b}^{n} \cdot \frac{\sqrt{\tilde{r}_{k}^{n *}\left(\rho \cdot b_{k}^{n *}\right) \cdot \sum_{n^{\prime}=1, n^{\prime} \neq n}^{N} b_{k}^{n^{\prime} *}}}{\sqrt{\frac{v^{n *}}{w^{n}} \cdot B}} .
\end{aligned}
$$

Recalling (2), (12), (18) and (21), variables $r_{k}^{n *}, \bar{r}_{k}^{n *}, b^{n}$, and $\tilde{b^{n}}$ in (52) are calculated as below.

$$
\begin{aligned}
& r_{k}^{n *}=r_{k}^{n *}\left(b_{k}^{n *}\right) \cdot \sum_{n^{\prime}=1, n^{\prime} \neq n}^{N} b_{k}^{n^{\prime} *}=\frac{v^{n *}}{w^{n}}\left(\sum_{n^{\prime \prime}=1}^{N} b_{k}^{n^{\prime \prime} *}\right)^{2} \Rightarrow \\
& \sqrt{r_{k}^{n *}\left(b_{k}^{n *}\right) \cdot \sum_{n^{\prime}=1, n^{\prime} \neq n}^{N} b_{k}^{n^{\prime *}}}=\sqrt{\frac{v^{n *}}{w^{n}}} \sum_{n^{\prime \prime}=1}^{N} b_{k}^{n^{\prime \prime} *}=\sqrt{\frac{v^{n *}}{w^{n}}} \cdot b_{k} \Rightarrow \\
& \sum_{k=1}^{K} \sqrt{r_{k}^{n *}\left(b_{k}^{n *}\right) \cdot \sum_{n^{\prime}=1, n^{\prime} \neq n}^{N} b_{k}^{n^{\prime *}}}=\sqrt{\frac{v^{n *}}{w^{n}}} \sum_{k=1}^{K} b_{k}=\sqrt{\frac{v^{n *}}{w^{n}}} \cdot B \text {. } \\
& \tilde{r}_{k}^{n *}\left(\rho \cdot b_{k}^{n *}\right) \cdot \sum_{n^{\prime}=1, n^{\prime} \neq n}^{N} b_{k}^{n^{\prime} *}=\frac{v^{n *}}{w^{n}}\left(\sum_{n^{\prime \prime}=1}^{N} b_{k}^{n^{\prime \prime} *}\right)^{2} \Rightarrow \\
& \sqrt{\tilde{r}_{k}^{n *}\left(\rho \cdot b_{k}^{n *}\right) \cdot \sum_{n^{\prime}=1, n^{\prime} \neq n}^{N}\left(b_{k}^{n^{\prime} *}\right)}=\sqrt{\frac{v^{n *}}{w^{n}}} \cdot b_{k} \Rightarrow \\
& \sum_{k=1}^{K} \sqrt{\tilde{r}_{k}^{n *}\left(\rho \cdot b_{k}^{n *}\right) \cdot \sum_{n^{\prime}=1, n^{\prime} \neq n}^{N}\left(b_{k}^{n^{\prime *}}\right)}=\sqrt{\frac{v^{n *}}{w^{n}}} \cdot B . \\
& b^{n}=\sum_{k=1}^{K} b_{k}^{n *} \text { and } \tilde{b}^{n}=\sum_{k^{\prime}=1, k^{\prime} \neq k}^{K} b_{k^{\prime}}^{n *}+\rho b_{k}^{n *} \\
& =\sum_{k^{\prime}=1}^{K} b_{k^{\prime}}^{n *}-b_{k}^{n *}+\rho \cdot b_{k}^{n *}=\left(b^{n}+(\rho-1) b_{k}^{n *}\right) \text {. }
\end{aligned}
$$

By substituting (53), (54) and (55) into (52) we obtain

$$
\begin{aligned}
\boldsymbol{f}\left(\mathbf{b}_{k}^{*}\right. & (t))-\boldsymbol{f}\left(\tilde{\mathbf{b}}_{k}^{*}(t)\right) \\
& =b^{n} \cdot \frac{\sqrt{\frac{v^{n *}}{w^{n}}} \cdot b_{k}}{\sqrt{\frac{v^{n *}}{w^{n}}} \cdot B}-\left(b^{n}+(\rho-1) \cdot b_{k}^{n *}\right) \cdot \frac{\sqrt{\frac{v^{n *} w^{n}}{w^{n}}} b_{k}}{\sqrt{\frac{v^{n *}}{w^{n}}} \cdot B} \\
& =b_{k}^{n *} \cdot \frac{b_{k}}{B} \cdot(1-\rho)>0,
\end{aligned}
$$

and, hence, $\boldsymbol{f}\left(\mathbf{b}_{k}^{*}(t)\right)$ is monotonous since $\boldsymbol{f}\left(\mathbf{b}_{k}^{*}(t)\right)>$ $\boldsymbol{f}\left(\tilde{\mathbf{b}}_{k}^{*}(t)\right)$ for $\mathbf{b}_{k}^{*}(t)>\tilde{\mathbf{b}}_{k}^{*}(t)$.

(iii) Scalability: For $\rho>1$, we denote the bandwidth allocation vector of user $k$ as $\hat{\mathbf{b}}_{k}^{*}(t)=\left(b_{k}^{1 *}, b_{k}^{2 *}, \ldots, \rho \cdot b_{k}^{n *}, \ldots, b_{k}^{N *}\right)$ and write the derivation of its winning strategy as

$$
\begin{aligned}
\rho \cdot \boldsymbol{f}\left(\mathbf{b}_{k}^{*}(t)\right) & -\boldsymbol{f}\left(\hat{\mathbf{b}}_{k}^{*}(t)\right)=\rho \cdot b^{n} \cdot \frac{\sqrt{r_{k}^{n *} \cdot \sum_{n=1, n \neq n}^{N} b_{k}^{n^{\prime \prime *}}}}{\sqrt{\frac{v^{n *}}{w^{n}}} B} \\
& -\hat{b}^{n} \cdot \frac{\sqrt{r_{k}^{n *}\left(\rho \cdot b_{k}^{n *}\right) \cdot \sum_{n^{\prime}=1, n^{\prime} \neq n}^{N} b_{k}^{n^{\prime *}}}}{\sqrt{\frac{v^{n \star}}{w^{n}}} B},
\end{aligned}
$$

where $b^{n}=\sum_{k=1}^{K} b_{k}^{n *}=\sum_{k^{\prime}=1, k^{\prime} \neq k}^{K} b_{k^{\prime}}^{n *}+b_{k}^{n *}$ and $\hat{b}^{n}=\sum_{k^{\prime}=1, k^{\prime} \neq k}^{K} b_{k^{\prime}}^{n *}+\rho \cdot b_{k}^{n *}$. Therefore, $\rho \cdot b^{n}-\hat{b}^{n}=$ $\sum_{k^{\prime}=1, k^{\prime} \neq k}^{K}\left(b_{k^{\prime}}^{n *} \cdot(\rho-1)\right)$ and $\tilde{r}_{k}^{n *}\left(\rho \cdot b_{k}^{n *}\right) \cdot \sum_{n^{\prime}=1, n^{\prime} \neq n}^{N} b_{k}^{n^{\prime} *}=$ $\frac{v^{n *}}{w^{n}} \cdot\left(\sum_{n^{\prime \prime}=1}^{N} b_{k}^{n^{\prime \prime} *}\right)^{2} \Rightarrow \sqrt{\tilde{r}_{k}^{n *}\left(\rho \cdot b_{k}^{n *}\right) \cdot \sum_{n^{\prime}=1, n^{\prime} \neq n}^{N}\left(b_{k}^{n^{\prime} *}\right)}=$ $\sqrt{\frac{v^{n *}}{w^{n}}} \cdot b_{k}$, which recalculates $(57)$ as $\rho \cdot \boldsymbol{f}\left(\mathbf{b}_{k}^{*}(t)\right)-\boldsymbol{f}\left(\hat{\mathbf{b}}_{k}^{*}(t)\right)=$ $\rho \cdot b^{n} \cdot \frac{\sqrt{\frac{v^{n *}}{w^{n}}} b_{k}}{\sqrt{\frac{v^{n *}}{w^{n}}} B}-\left(\hat{b}^{n}\right) \cdot \frac{\sqrt{\frac{v^{n *}}{w^{n}}} b_{k}}{\sqrt{\frac{v^{n *}}{w^{n}}} B}=\frac{b_{k}}{B} \cdot\left(\rho \cdot b^{n}-\hat{b}^{n}\right)=\frac{b_{k}}{B} \cdot$ $\sum_{k^{\prime}=1, k^{\prime} \neq k}^{K} b_{k^{\prime}}^{n *}(\rho-1)>0$, and yields that $\boldsymbol{f}\left(\mathbf{b}_{k}^{*}(t)\right)$ is scalable as $\rho \cdot \boldsymbol{f}\left(\mathbf{b}_{k}^{*}(t)\right)>\boldsymbol{f}\left(\hat{\mathbf{b}}_{k}^{*}(t)\right)$. This completes the proof of Proposition 2.

\section{APPENDIX E}

PROOFS OF PROPOSITION 3 AND PROPOSITION 4

The set $C\left(g_{i}\left(y_{k}^{n}, t\right)\right)$ in (14) includes variables $\left(y_{1}^{1}, y_{1}^{2}, \ldots, y_{1}^{N}, \ldots, y_{k}^{1}, \ldots, y_{k}^{N}, \ldots, y_{K}^{1}, \ldots, y_{K}^{N}, t\right)$, which are $((K \cdot N)+1)$ in total, i.e., $(K \cdot N)$ variables for $y_{k}^{n}$ in (C1)-(C3) plus one variable for $t$ in (C4). Also, the Jacobian matrix $G\left(g_{i}\left(y_{k}^{n}, t\right)\right)$ in (15) can be represented by the partial derivatives of each of the $\left\{g_{i}\left(y_{k}^{n}, t\right)\right\}$ constraints w.r.t $y_{k}^{n}$ and $t$, as shown in (58) at the top of this page. From (58) the Jacobian matrix is computed as $G\left(g_{i}\left(y_{k}^{n}, t\right)\right)=\left[\nabla g_{1}\left(y_{k}^{n}, t\right) \ldots \nabla g_{K+2}\left(y_{k}^{n}, t\right)\right]^{T}=\mathbf{J c b}$, where Jcb is $K+2$ rank matrix given in (59) at the bottom of this page. Also, for $N>1$ the number of variables (i.e. $(K \cdot N)+1)$ in $G\left(g_{i}\left(y_{k}^{n}, t\right)\right)$ is larger than the number of constraints (i.e. $K+2$ ) therefore, there exists a nonzero vector $\boldsymbol{\psi}=\left[\psi_{1}^{1} \ldots \psi_{1}^{N} \psi_{2}^{1} \ldots \psi_{2}^{N} \ldots \psi_{K}^{1} \ldots \psi_{K}^{N} \psi_{K N+1}\right]^{T}$ to resolve $G\left(g_{i}\left(y_{k}^{n}, t\right)\right)$ by $G\left(g_{i}\left(y_{k}^{n}, t\right)\right) \cdot \boldsymbol{\psi}^{T}=0$ with $\psi_{k}^{1}=\left(\frac{\nabla g_{k}^{2}}{\nabla g_{k+1}^{1}}\right)\left(\frac{\nabla g_{k+1}^{1}-\nabla g_{k+1}^{2}}{\nabla g_{k}^{1}-\nabla g_{k}^{2}}\right), \psi_{k}^{2}=-\left(\frac{\nabla g_{k}^{1}}{\nabla g_{k+1}^{1}}\right)\left(\frac{\nabla g_{k+1}^{1}-\nabla g_{k+1}^{2}}{\nabla g_{k}^{1}-\nabla g_{k}^{2}}\right)$, $\psi_{k}^{3}=0, \ldots, \psi_{k}^{N}=0$ for $k=1,3,5, \ldots$, and $\psi_{k}^{1}=-\frac{\nabla g_{k 2}}{\nabla g_{k 1}}$, $\psi_{k}^{2}=1, \psi_{k}^{3}=0, \ldots, \psi_{k}^{N}=0, \psi_{K N+1}=0$ for $k=2,4,6, \ldots$ This completes the proof of Proposition 3. Furthermore, to show that constraints $\left\{g_{i}\left(y_{k}^{n}, t\right)\right\}, i=0, \ldots, K+1$ are continuous and twice differentiable in $\mathbb{R}^{(K \cdot N)+1}$, it is sufficient to prove that the Hessian matrix $H\left(g_{i}\left(y_{k}^{n}, t\right)\right)=$ $\nabla_{y^{*}, t^{*}}^{2} \boldsymbol{L}\left(\boldsymbol{y}^{*}, t^{*}, \lambda^{*}, \boldsymbol{\xi}^{*}, \mu^{*}\right)$ of $\boldsymbol{L}\left(\boldsymbol{y}^{*}, t^{*}, \lambda^{*}, \boldsymbol{\xi}^{*}, \mu^{*}\right)$ in (31) is negative definite, i.e., $\boldsymbol{\psi}^{T} \cdot \nabla_{y^{*}, t^{*}}^{*} \boldsymbol{L}\left(\boldsymbol{y}^{*}, t^{*}, \lambda^{*}, \boldsymbol{\xi}^{*}, \mu^{*}\right)$. $\boldsymbol{\psi}<0$. Indeed, we calculate the second derivatives of $\boldsymbol{L}$ as $\frac{\partial^{2} L}{\partial y_{k}^{n 2}}=-\frac{\left(\eta_{0}+\xi_{k}^{*}\right) \cdot b_{k}^{n *}}{\ln 2} \cdot \frac{\left(\frac{\left|h_{k}^{n}\right|^{2}}{b_{k}^{n *} \cdot N_{0}}\right)^{2}}{\left(1+\frac{y_{k}^{n *}}{t^{*}} \cdot \frac{\left|h_{k}^{n}\right|^{2}}{b_{k}^{n *} \cdot N_{0}}\right)^{2}}<0, \frac{\partial^{2} L}{\partial t^{2}}=$

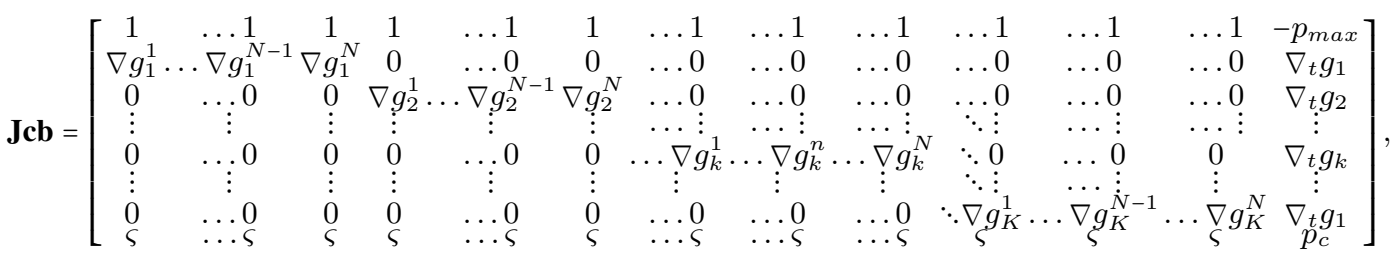


$-\sum_{k=1}^{K} \sum_{n=1}^{N}\left(\eta_{0}+\xi_{k}^{*}\right) \cdot \frac{b_{k}^{n *}}{t^{*} \cdot \ln 2} \cdot \frac{\left(\frac{\left.y_{k}^{n *} \cdot \frac{\left.h_{k}^{n}\right|^{2}}{t^{*}}\right)_{k}^{n *} N_{0}}{2}\right.}{\left(1+\frac{y_{k}^{n *}}{t^{*}} \cdot \frac{\left|h_{k}^{n}\right|^{2}}{b_{k}^{n *} \cdot N_{0}}\right)^{2}}<0, \frac{\partial^{2} L}{\partial y_{k}^{n} \partial y_{k}^{m}}=$
$\frac{\partial^{2} L}{\partial y_{k}^{m} \partial y_{k}^{n}}=0, m \neq n, \frac{\partial^{2} L}{\partial y_{k}^{n *} \partial t^{*}}=\frac{\partial^{2} L}{\partial t \partial y_{k}^{n}}=\frac{\left(\eta_{0}+\xi_{k}^{*}\right) \cdot b_{k}^{n *}}{\ln 2} \cdot$
$\frac{\left(\frac{y_{k}^{n *}}{t^{*}} \cdot \frac{\left|h_{k}^{n}\right|^{2}}{b_{k}^{n *} \cdot N_{0}}\right)^{2}}{\left(1+\frac{y_{k}^{n *}}{t^{*}} \cdot \frac{\left|h_{k}^{n}\right|^{2}}{b_{k}^{n *} \cdot N_{0}}\right)^{2}}>0$ hence, $\boldsymbol{L}$ is twice differentiable. In addition, due to the structure of $\psi$, the multiplications $\psi^{T}$. $\nabla_{y^{*}, t^{*}}^{*} \boldsymbol{L}\left(\boldsymbol{y}^{*}, t^{*}, \lambda^{*}, \boldsymbol{\xi}^{*}, \mu^{*}\right) \cdot \boldsymbol{\psi}$ depend on the non-zero elements of $\boldsymbol{\psi}$ that correspond to the diagonal of $H\left(g_{i}\left(y_{k}^{n}, t\right)\right)$ computed by

$$
\begin{aligned}
\left(\frac{\nabla g_{1}^{2}}{\nabla g_{1}^{1}}\right)^{2} \cdot\left(\frac{\nabla g_{2}^{1}}{\nabla g_{1}^{1}}\right)^{2} \cdot\left(\frac{\nabla g_{2}^{1}-\nabla g_{2}^{2}}{\nabla g_{1}^{1}-\nabla g_{1}^{2}}\right)^{2} \cdot \frac{\partial^{2} L}{\partial y_{1}^{12}} & \\
+\frac{\partial^{2} L}{\partial y_{1}^{22}}+ & \left(\frac{\nabla g_{2}^{2}}{\nabla g_{2}^{1}}\right)^{2} \cdot \frac{\partial^{2} L}{\partial y_{2}^{12}}+\frac{\partial^{2} L}{\partial y_{2}^{22}} \\
+\cdots & +\left(\frac{\nabla g_{3}^{2}}{\nabla g_{3}^{1}}\right)^{2} \cdot\left(\frac{\nabla g_{4}^{1}}{\nabla g_{3}^{1}}\right)^{2} \cdot\left(\frac{\nabla g_{4}^{1}-\nabla g_{4}^{2}}{\nabla g_{3}^{1}-\nabla g_{3}^{2}}\right)^{2} \cdot \frac{\partial^{2} L}{\partial y_{3}^{12}}+\frac{\partial^{2} L}{\partial y_{3}^{22}} \\
& +\ldots+\left(\frac{\nabla g_{K}^{2}}{\nabla g_{K}^{1}}\right)^{2} \cdot \frac{\partial^{2} L}{\partial y_{K}^{12}}+\frac{\partial^{2} L}{\partial y_{K}^{2}}<0
\end{aligned}
$$

From (60) and recalling that $\frac{\partial^{2} L}{\partial y_{k}^{n 2}}<0$ for all $n$ and $k$, the Hessian $H\left(g_{i}\left(y_{k}^{n}, t\right)\right)$ of $\boldsymbol{L}\left(\boldsymbol{y}^{*}, t^{*}, \lambda^{*}, \boldsymbol{\xi}^{*}, \mu^{*}\right)$ is negative definite. This completes the proof of Proposition 4

\section{REFERENCES}

[1] Q. Ni and C. C. Zarakovitis, "Nash Bargaining Game Theoretic Scheduling for Joint Channel and Power Allocation in Cognitive Radio Systems," IEEE J. Sel. Areas Commun., vol. 30, no. 1, pp. 70-81, Jan 2012

[2] E. Borel, "The theory of play and integral equations with skew symmetric kernels" Econometrica, vol 21 pp. 97-100, 1953

[3] E. M. L. Beale and G. P. N. Heselden, "An Approximate Method of Solving Blotto Games", Naval Research Logistics Quarterly, vol. 9, no. 2 pp. $65-80,1962$

[4] J. Duffy and A. Matros, "Stochastic Asymmetric Blotto Games: An Experimental Study", J. Economic Behavior \& Organization, vol. 139, pp. 88-105, Jul 2017

[5] A. Ahmadinejad, S. Dehghani, M. T. Hajiaghayi, B. Lucier, H. Mahini and S. Seddighin, "From Duels to Battlefields: Computing Equilibria of Blotto and other Games", Proc. of the Thirtieth AAAI Conf. on Artificial Intelligence, Phoenix, Arizona, pp. 369-375, 2016

[6] C. Tan, T. Chuah and S. Tan, "Fair Subcarrier and Power Allocation for Multiuser Orthogonal Frequency-Division Multiple Access Cognitive Radio Networks using Blotto Game," IET J. Commun., vol. 5, no. 11, pp. 1607-1618, 22 Jul 2011

[7] C. K. Tan, M. L. Sim, T. C. Chuah and S. W. Tan, "Blotto GameBased Low-Complexity Fair Multiuser Subcarrier Allocation for Uplink OFDMA Networks," EURASIP J. Wireless Commun. Network, Aug 2011

[8] D. Tian, J. Zhou, Z. Sheng, M. Chen, Q. Ni and V. C. M. Leung, "SelfOrganized Relay Selection for Cooperative Transmission in Vehicular Ad-Hoc Networks," IEEE Trans. Veh. Technol., vol. 66, no. 10, pp. 9534-9549, Oct 2017

[9] S. Zhao, Y. Yang, Z. Shao, X. Yang, H. Qian and C. Wang, "FEMOS: Fog-Enabled Multitier Operations Scheduling in Dynamic Wireless Networks", IEEE J. Internet of Things, vol. 5, no. 2, pp. 1169-1183, Apr 2018

[10] A. S. Ghazy, H. A. I. Selmy and H. M. H. Shalaby, "Fair resource allocation schemes for cooperative dynamic free-space optical networks", IEEE/OSA J. Optical Commun. and Networking, vol. 8, no. 11, pp. 822834, Nov 2016

[11] D. M. Blough, G. Resta and P. Santi, "Interference-aware time-based fairness for multihop wireless networks," IEEE INFOCOM, pp. 1-9, San Francisco, CA, 2016

[12] J. Du, L. Zhao, J. Feng and X. Chu, "Computation Offloading and Resource Allocation in Mixed Fog/Cloud Computing Systems With Min-Max Fairness Guarantee", IEEE Trans. Commun., vol. 66, no. 4, pp. 1594-1608, Apr 2018

[13] "Project Fi", [Online]: http://fi.google.com/

[14] M. Hajimirsaadeghi and N. B. Mandayam, "A Dynamic Colonel Blotto Game Model for Spectrum Sharing in Wireless Networks," Annual Conf.
Commun., Control, and Computing (Allerton), Monticello, IL, pp. $287-$ 294, 2017

[15] P. Barelli, S. Govindan, and R. Wilson, "Competition for a Majority", Econometrica, vol. 82, no.1, pp. 271-314, Jan 2014

[16] G. Schwartz, P. Loiseau, and S. S. Sastry, "The Heterogeneous Colonel Blotto Game", Int. Conf. on Network Games, Control and Optimization (NetGCoop), Trento, Italy, pp. 232-238, 2014

[17] A. Charnes and W. W. Cooper, "Systems Evaluation and Repricing Theorems," O.N.R. Memorandum, vol. 31, Sep 1960

[18] M. Dziubinski, "Non-symmetric General Lotto games", Int. J. of Game Theory, vol. 42, no. 4, pp. 801-833, Nov 2013

[19] S. Ha and Y. Han, "A Competitive Fair Subchannel Allocation for OFDMA System using an Auction Algorithm," IEEE Proc. Veh. Tech. Conf. (VTC'07 Fall), Baltimore, USA, Sep 2007

[20] A. Ferdowsi, A. Sanjab, W. Saad, and T. Basar, "Generalized Colonel Blotto game", IEEE American Control Conference, Milwaukee, WI, USA, Jun 2018

[21] G. Yu, Q. Chen, R. Yin, H. Zhang and G. Y. Li, "Joint Downlink and Uplink Resource Allocation for Energy-Efficient Carrier Aggregation," IEEE Trans. Wireless Commun., vol. 14, no. 6, pp. 3207-3218, Jun 2015

[22] M. L. Tham, S. F. Chien, D. W. Holtby and S. Alimov, "Energy-Efficient Power Allocation for Distributed Antenna Systems With Proportional Fairness," IEEE Trans. Green Commun. Networking, vol. 1, no. 2, pp. 145-157, Jun 2017

[23] Q. Chen, G. Yu, R. Yin and G. Y. Li, "Energy-Efficient User Association and Resource Allocation for Multistream Carrier Aggregation," IEEE Trans. Veh. Technol., vol. 65, no. 8, pp. 6366-6376, Aug 2016

[24] X. Ge, X. Huang, Y. Wang, M. Chen, Q. Li, T. Han and C. X. Wang, "Energy Efficiency Optimization for MIMO-OFDM Mobile Multimedia Communication Systems with QoS Constraints," IEEE Trans. Veh. Technol., vol. 63, no. 5, p. 2127-2138, Jun 2014

[25] D. W. K. Ng, E. S. Lo and R. Schober, "Energy-efficient Resource Allocation in Multi-cell OFDMA Systems with Limited Backhaul Capacity", IEEE Trans. Wireless Commun., vol. 10, no. 11, p. 3618-3631, 2012

[26] X. Xiao, X. Tao and J. Lu, "QoS-Aware Energy-Efficient Radio Resource Scheduling in Multi-User OFDMA Systems", IEEE Commun. Lett., vol. 17, pp. 75-78, Jan 2013

[27] C. C. Zarakovitis, Q. Ni and J. Spiliotis, "Energy Efficient Green Wireless Communication Systems with Imperfect CSI and Data Outage," IEEE J. Sel. Areas Commun., vol. 34, no. 12, pp. 3108 - 3126, Dec 2016

[28] X. Wang, F. Zheng and P. X. Zhu, "Energy-Efficient Resource Allocation in Coordinated Downlink Multi-cell OFDMA Systems," IEEE Trans. Veh. Tech., vol. 65, no. 3, pp. 1395-1408, Mar 2016

[29] W. Xu, Y. Cui, H. Zhang, G. Y. Li and X. You, "Robust Beamforming With Partial Channel State Information for Energy Efficient Networks," IEEE J. Sel. Areas Commun., vol. 33, no. 12, p. 2920-2935, Dec 2015

[30] E. Bedeer, O. Amin, O. A. Dobre, M. H. Ahmed and K. E. Baddour, "Energy-Efficient Power Loading for OFDM-Based Cognitive Radio Systems With Channel Uncertainties," IEEE Trans. Vech. Technol., vol. 64, no. 6, p. 2672-2677, Jun 2015

[31] S. Boyd and L. Vandenberghe, "Convex Optimization", Cambridge Univ Press, 2004

[32] R. Jain, D. Chi and W. Hawe, "A Quantitative Measure of Fairness and Discrimination for Resource Allocation in Shared Systems," DEC Research Report TR-301, 1984

[33] C. P. Simon, L. E. Blume, "Mathematics for Economists", W. W. Norton and Company, Inc., New York, USA, 2010

[34] Z. Han, D. Niyato, W. Saad, T. Başar, A Hjorungnes, "Game Theory in Wireless and Communication Networks: Theory, Models, and Applications", Cambridge University Press, 2011

[35] J. M. Ortega and W. C. Rheinboldt, "Iterative Solution of Nonlinear Equations in Several Variables", New York, 1970

[36] R. M. Corless, G. H. Gonnet and D. E. Knuth, "On the Lambert W Function," J. Adv. Compu. Math., pp. 329-359, 1999

[37] Andrzej Granas and James Dugundji, "Fixed Point Theory", SpringerVerlag, New York, 2003

[38] S. Banach, "Sur les Operations dans les Ensembles Abstraits et leur Application aux Equations Integrales", Fund. Math., vol 3, pp. 133-181, 1922

[39] J. B. Rosen, "Existence and Uniqueness of Nash Equilibrium Points for Concave n-person Games", Econometrica, vol. 33, pp. 520-534, 1965 